\title{
On the continuity of Hausdorff dimension of Julia sets and similarity between the Mandelbrot set and Julia sets
}

by

\author{
Juan Rivera-Letelier (Stony Brook, NY)
}

\begin{abstract}
Given $d \geq 2$ consider the family of polynomials $P_{c}(z)=z^{d}+c$ for $c \in \mathbb{C}$. Denote by $J_{c}$ the Julia set of $P_{c}$ and let $\mathcal{M}_{d}=\left\{c \mid J_{c}\right.$ is connected $\}$ be the connectedness locus; for $d=2$ it is called the Mandelbrot set. We study semihyperbolic parameters $c_{0} \in \partial \mathcal{M}_{d}$ : those for which the critical point 0 is not recurrent by $P_{c_{0}}$ and without parabolic cycles. The Hausdorff dimension of $J_{c}$, denoted by $\operatorname{HD}\left(J_{c}\right)$, does not depend continuously on $c$ at such $c_{0} \in \partial \mathcal{M}_{d}$; on the other hand the function $c \mapsto \operatorname{HD}\left(J_{c}\right)$ is analytic in $\mathbb{C}-\mathcal{M}_{d}$. Our first result asserts that there is still some continuity of the Hausdorff dimension if one approaches $c_{0}$ in a "good" way: there is $C=C\left(c_{0}\right)>0$ such that for a sequence $c_{n} \rightarrow c_{0}$,

$$
\text { if } \operatorname{dist}\left(c_{n}, \mathcal{M}_{d}\right) \geq C\left|c_{n}-c_{0}\right|^{1+1 / d}, \quad \text { then } \quad \operatorname{HD}\left(J_{c_{n}}\right) \rightarrow \operatorname{HD}\left(J_{c_{0}}\right) .
$$
\end{abstract}

To prove this we use the fact that $\mathcal{M}_{d}$ and $J_{c_{0}}$ are similar near $c_{0}$. In fact we prove that the biholomorphism $\psi: \overline{\mathbb{C}}-J_{c_{0}} \rightarrow \overline{\mathbb{C}}-\mathcal{M}_{d}$ tangent to the identity at infinity is conformal at $c_{0}$ : there is $\lambda \neq 0$ such that

$$
\psi(w)=c_{0}+\lambda\left(w-c_{0}\right)+\mathcal{O}\left(\left|w-c_{0}\right|^{1+1 / d}\right) \quad \text { for } w \notin J_{c_{0}} .
$$

This implies that the local structures of $\mathcal{M}_{d}$ and $J_{c_{0}}$ at $c_{0}$ are similar. The fact that $\lambda \neq 0$ is related to a transversality phenomenon that is well known for Misiurewicz parameters and that we extend to the semihyperbolic case. We also prove that for some $C>0$,

$$
d_{\mathrm{H}}\left(J_{c}, J_{c_{0}}\right) \leq C\left|c-c_{0}\right|^{1 / d} \text { and } d_{\mathrm{H}}\left(K_{c}, J_{c_{0}}\right) \leq C\left|c-c_{0}\right|^{1 / d}
$$

where $d_{\mathrm{H}}$ denotes the Hausdorff distance.

1. Introduction. Given $d \geq 2$ consider the family of monic polynomials $P_{c}(z)=z^{d}+c$, for $c \in \mathbb{C}$, whose unique finite critical point is 0 . The set

$$
K_{c}=\left\{z \in \mathbb{C} \mid\left\{P_{c}^{n}(z)\right\}_{n \geq 0} \text { is bounded }\right\}
$$

is called the filled-in Julia set of $P_{c}$ and $J_{c}=\partial K_{c}$ is called the Julia set of $P_{c}$.

2000 Mathematics Subject Classification: 37F45, 37D25.

Most of this work was done at Université de Paris Sud and it was partially supported by "Fundacion Andes" and a "Beca Presidente de la Republica", Chile. 
We study dynamics of polynomials $P_{c}$ such that the critical point 0 is not recurrent and $0 \in J_{c}$. These polynomials are semihyperbolic in the sense of [CJY]: a semihyperbolic polynomial $P_{c_{0}}$ is either as above or it is hyperbolic. The latter means that either $0 \notin K_{c_{0}}$ or $P_{c_{0}}$ has an attracting cycle. Dynamics of hyperbolic polynomials is well understood, so we only consider semihyperbolic polynomials that are not hyperbolic.

Examples of semihyperbolic polynomials are Misiurewicz polynomials: a polynomial $P_{c_{0}}$ is said to be Misiurewicz if the critical point of $P_{c_{0}}$ is strictly preperiodic. In this case $P_{c_{0}}$ is not hyperbolic; see [CG]. The set of parameters $c \in \mathbb{C}$ for which $P_{c}$ is Misiurewicz is countable; see [DH2]. The set of parameters $c \in \mathbb{C}$ for which $P_{c}$ is semihyperbolic but not hyperbolic, is much larger: Shishikura proved in [Sh] that it has Hausdorff dimension two.

It follows from a theorem of Fatou that the Julia set $J_{c}$ is connected if and only if $c \in K_{c}$; see [CG]. Consider the connectedness locus

$$
\mathcal{M}_{d}=\left\{c \in \mathbb{C} \mid J_{c} \text { is connected }\right\}=\left\{c \in \mathbb{C} \mid c \in K_{c}\right\} .
$$

If $d=2$ this set is also denoted by $\mathcal{M}$ and is called the Mandelbrot set. It is known that $\mathcal{M}_{d}$ is compact and connected and moreover $\mathbb{C}-\mathcal{M}_{d}$ is homeomorphic to $\{|z|>1\}$; see [DH2].

If $P_{c_{0}}$ is semihyperbolic, then $c_{0} \in \partial \mathcal{M}_{d}$ if and only $P_{c_{0}}$ is not hyperbolic; see [DH2].

1.1. On the continuity of Hausdorff dimension. Our first result is about the dependence of the Hausdorff dimension of Julia sets on the parameter; see also $[\mathrm{McM}],[\mathrm{DSZ}],[\mathrm{GSm}],[\mathrm{GSw}]$ and [UZ]. We denote the Hausdorff dimension by HD.

The Hausdorff dimension of the Julia set varies in an analytic way in the exterior of $\mathcal{M}_{d}$; see $[\mathrm{R}]$. On the other hand Shishikura proved in [Sh] that there is a residual (hence dense) set of parameters in $\partial \mathcal{M}_{d}$ for which $\operatorname{HD}\left(J_{c}\right)=2$ and by $[\mathrm{U}]$ we have $\operatorname{HD}\left(J_{c_{0}}\right)<2$ for any $c_{0} \in \partial \mathcal{M}_{d}$ such that $P_{c_{0}}$ is semihyperbolic. So the Hausdorff dimension of the Julia set does not vary continuously in the parameter at such $c_{0}$.

The following theorem asserts that there is some continuity of the Hausdorff dimension at $c_{0} \in \partial \mathcal{M}_{d}$ such that $P_{c_{0}}$ is semihyperbolic, if one approaches $c_{0}$ from the exterior of $\mathcal{M}_{d}$ in a "good" way.

Theorem A. Let $c_{0} \in \partial \mathcal{M}_{d}$ be such that $P_{c_{0}}$ is semihyperbolic. Then there is some $C>0$ such that if a sequence $c_{n} \rightarrow c_{0}$ is such that

$$
\operatorname{dist}\left(c_{n}, \mathcal{M}_{d}\right) \geq C\left|c_{n}-c_{0}\right|^{1+1 / d},
$$

then $\operatorname{HD}\left(J_{c_{n}}\right) \rightarrow \operatorname{HD}\left(J_{c_{0}}\right)$.

As we will see below this is much stronger than radial continuity of the Hausdorff dimension; see Figure 1. 


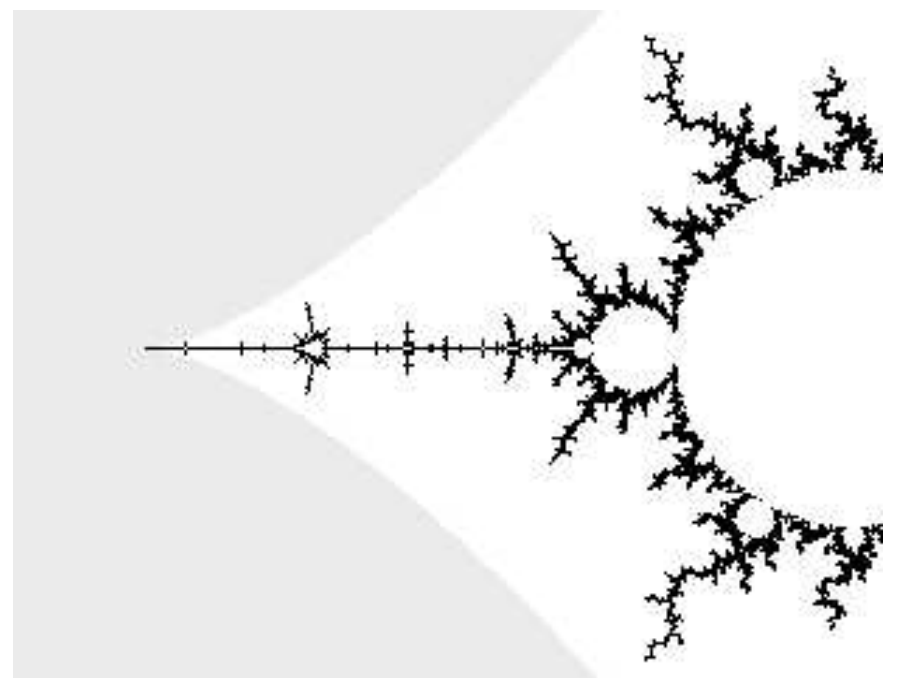

Fig. 1. Detail of the Mandelbrot set about the Misiurewicz parameter -2 . The Hausdorff dimension of Julia sets corresponding to a sequence of parameters in the grey part converging to -2 , should converge to $\operatorname{HD}\left(J_{-2}\right)=1$.

1.2. Similarities between the Mandelbrot set and Julia sets. One of the main ingredients in the proof of Theorem $\mathrm{A}$ is the similarity of $\mathcal{M}_{d}$ and $J_{c_{0}}$ at a parameter $c_{0} \in \partial \mathcal{M}_{d}$ such that $P_{c_{0}}$ is semihyperbolic.

There is an heuristic principle that the local structure in the dynamical plane should be similar to the structure in the parameter plane, at least at parameters with some expanding property. For example Shishikura made use of this principle in proving that the boundary of the Mandelbrot set has Hausdorff dimension two; see [Sh]. Also Wenstorm in [W] proved some remarkable similarities between the Mandelbrot set and the Fibonacci Julia set; see also [R-L1].

In [L] T. Lei proved that for a Misiurewicz parameter $c_{0}$, the sets $\mathcal{M}$ and $J_{c_{0}}$ are asymptotically similar at $c_{0}$; see also [R-L1] and [R-L2]. To define this notion let us consider the following definitions. Given a compact subset $\mathrm{X}$ of $\mathbb{C}$ and $r>0$, let

$$
X_{r}=\left(\left\{r^{-1} w \mid w \in X\right\} \cap \mathbb{D}\right) \cup \partial \mathbb{D} .
$$

That is, to construct $X_{r}$ consider the intersection of $X$ with the disc of radius $r$ centered at 0 , scale it to the unit disk and for technical reasons add $\partial \mathbb{D}$. Moreover, for $\lambda \in \mathbb{C}-\{0\}$ and $\zeta \in \mathbb{C}$ we define $\lambda X=\{\lambda w \mid w \in X\}$ and $X-\zeta=\{w-\zeta \mid w \in X\}$.

Theorem (T. Lei $[\mathrm{L}])$. Consider $c_{0} \in \mathcal{M}$ such that $P_{c_{0}}$ is Misiurewicz. Then there is $\lambda \in \mathbb{C}-\{0\}$ such that 


$$
\lim _{r \rightarrow 0} d_{\mathrm{H}}\left(\left(\mathcal{M}-c_{0}\right)_{r},\left(\lambda\left(J_{c_{0}}-c_{0}\right)\right)_{r}\right)=0,
$$

where $d_{\mathrm{H}}$ denotes the Hausdorff distance.

See also [R-L1]. Whenever this holds, it is said that $\mathcal{M}_{d}$ and $J_{c_{0}}$ are asymptotically similar at $c_{0}$. We generalize this theorem to semihyperbolic parameters as an easy corollary to the following theorem.

Theorem B $\left(C^{1+1 / d}\right.$-conformality of external maps). Let $c_{0} \in \partial \mathcal{M}_{d}$ be such that $P_{c_{0}}$ is semihyperbolic, and let $\varphi_{c_{0}}: \overline{\mathbb{C}}-J_{c_{0}} \rightarrow \overline{\mathbb{C}}-\overline{\mathbb{D}}$ and $\varphi_{\mathcal{M}_{d}}: \overline{\mathbb{C}}-\mathcal{M}_{d} \rightarrow \overline{\mathbb{C}}-\overline{\mathbb{D}}$ be the canonical uniformizations (tangent to the identity at infinity). Then $\lambda=\sum_{n \geq 0} 1 /\left(P_{c_{0}}^{n}\right)^{\prime}\left(c_{0}\right) \in \mathbb{C}-\{0\}$ and

$$
\begin{array}{ll}
\varphi_{c_{0}}^{-1} \circ \varphi_{\mathcal{M}_{d}}(c)=c_{0}+\lambda\left(c-c_{0}\right)+\mathcal{O}\left(\left|c-c_{0}\right|^{1+1 / d}\right) & \text { for } c \notin \mathcal{M}_{d}, \\
\varphi_{\mathcal{M}_{d}}^{-1} \circ \varphi_{c_{0}}(w)=c_{0}+\lambda^{-1}\left(w-c_{0}\right)+\mathcal{O}\left(\left|w-c_{0}\right|^{1+1 / d}\right) & \text { for } w \notin J_{c_{0}} .
\end{array}
$$

The conformality of these maps is a finer notion of similarity; see [R-L1]. For Misiurewicz parameters the similarity factor, given by the series above, is essentially a geometric series and therefore it can be calculated explicitly. For example -2 is a quadratic Misiurewicz parameter and $\lambda=2 / 3$ in this case.

The following are corollaries of Theorem B, whose proofs are in Appendix 1. The following one improves T. Lei's theorem.

Corollary (Asymptotic similarity). Let $c_{0} \in \partial \mathcal{M}_{d}$ be such that $P_{c_{0}}$ is semihyperbolic. Then there is a constant $C>0$ such that for $r>0$ small,

$$
d_{\mathrm{H}}\left(\left(\mathcal{M}_{d}-c_{0}\right)_{r},\left(\lambda\left(J_{c_{0}}-c_{0}\right)\right)_{r}\right) \leq C r^{1 / d} .
$$

In particular $\mathcal{M}_{d}$ and $J_{c_{0}}$ are asymptotically similar at $c_{0}$.

COROLlary. Let $c_{0} \in \partial \mathcal{M}_{d}$ be such that $P_{c_{0}}$ is semihyperbolic and put $D=\operatorname{HD}\left(J_{c_{0}}\right)$. Then there is a constant $C>0$ such that

$$
\text { Lebesgue-measure }\left(\mathcal{M}_{d} \cap B_{r}\left(c_{0}\right)\right) \leq C r^{2+(2-D) / d} \text {. }
$$

Recall that by [U], $D=\operatorname{HD}\left(J_{c_{0}}\right)<2$ in this case, so these parameters are density points of the complement of $\mathcal{M}_{d}$. Combining Theorems $\mathrm{A}$ and $\mathrm{B}$ we obtain the following immediate corollary.

Corollary. Let $c_{0} \in \partial \mathcal{M}_{d}$ be such that $P_{c_{0}}$ is semihyperbolic and let $\lambda=\sum_{n \geq 0} 1 /\left(P_{c_{0}}^{n}\right)^{\prime}\left(c_{0}\right) \in \mathbb{C}-\{0\}$ be as in Theorem B. Then there is some $C>0$ such that if $z_{n} \rightarrow c_{0}$ with $\operatorname{dist}\left(z_{n}, J_{c_{0}}\right) \geq C\left|z_{n}-c_{0}\right|^{1+1 / d}$, then letting $c_{n}=c_{0}+\lambda\left(z_{n}-c_{0}\right)$ we have

$$
\operatorname{HD}\left(J_{c_{n}}\right) \rightarrow \operatorname{HD}\left(J_{c_{0}}\right)
$$

For example the polynomial $z^{2}-2$ is a quadratic Misiurewicz polynomial and its Julia set is the interval $[-2,2]$. So, by the previous corollary, there is a constant $C_{0}>0$ such that if a sequence $c_{n} \rightarrow c_{0}$ is at the left of the graph 
of the semicubical parabola $y=C_{0}(x+2)^{3 / 2}$, then $\operatorname{HD}\left(J_{c_{n}}\right) \rightarrow \operatorname{HD}\left(J_{c_{0}}\right)$; see Figure 1.

It follows by $[\mathrm{H}]$ that if $\zeta \in \partial \mathbb{D}$ is such that the $\operatorname{ray}\left\{\varphi_{\mathcal{M}_{d}}^{-1}(r \zeta) \mid r>1\right\}$ accumulates at $c_{0}$, then $\varphi_{\mathcal{M}_{d}}^{-1}$ extends continuously to $\zeta$, with $\varphi_{\mathcal{M}_{d}}^{-1}(\zeta)=c_{0}$. Recall that a Stolz angle in $\mathbb{C}-\overline{\mathbb{D}}$ at $\zeta$ is a set of the form

$$
\left\{r e^{i \theta} \zeta \mid r \geq 1 \text { and }|\theta| \leq C(r-1)\right\} \quad \text { for some } C>0 \text {. }
$$

The following corollary was obtained in $[\mathrm{BR}]$ for Misiurewicz parameters; see also [GSm] and [GSw]. It follows from the previous corollary together with the fact that $\mathbb{C}-J_{c_{0}}$ is a John domain; see [CJY] and Preliminaries.

Corollary (Angular convergence of Hausdorff dimension). Let $c_{0} \in$ $\partial \mathcal{M}_{d}$ be such that $P_{c_{0}}$ is semihyperbolic and let $\zeta \in \partial \mathbb{D}$ be such that $c_{0}=$ $\varphi_{\mathcal{M}_{d}}^{-1}(\zeta)$. Then the function

$$
w \mapsto \operatorname{HD}\left(J_{\varphi_{\mathcal{M}_{d}}^{-1}(w)}\right)
$$

is continuous in the closure of any Stolz angle in $\mathbb{C}-\overline{\mathbb{D}}$ at $\zeta$.

1.3. Stability of Julia sets. Our final result is about the stability of Julia sets under perturbation.

Theorem C. Let $c_{0} \in \partial \mathcal{M}_{d}$ be such that $P_{c_{0}}$ is semihyperbolic. Then there is $C>0$ such that for $c \in \mathbb{C}$ close to $c_{0}$,

$$
d_{\mathrm{H}}\left(J_{c_{0}}, J_{c}\right) \leq C\left|c-c_{0}\right|^{1 / d} \quad \text { and } \quad d_{\mathrm{H}}\left(J_{c_{0}}, K_{c}\right) \leq C\left|c-c_{0}\right|^{1 / d} .
$$

In particular the Julia set varies continuously with the parameter at semihyperbolic parameters. This is true for all parameters without parabolic or Siegel cycles; see [D].

We remark that this theorem is sharp, that is, $\mathcal{O}\left(\left|c-c_{0}\right|^{1 / d}\right)$ cannot be replaced by $o\left(\left|c-c_{0}\right|^{1 / d}\right)$; see end of Section 4.2.

1.4. Organization of the paper. It follows from a theorem of Mañé that for a semihyperbolic polynomial the set of accumulation points of the orbit of the critical point, denoted by $\omega(0)$, is a hyperbolic set. In Section 2 we construct a Markov partition for $\omega(0)$ with puzzles.

In Section 3 we prove that semihyperbolic polynomials have a property that we call Almost Uniform Expansion, which by [R-L3] is equivalent to the Collet-Eckmann condition. With this property we prove the Main Lemma about backward stability under perturbations (Section 3.1). This property is related to backward shadowing properties. Section 3 is independent of Section 2.

In Section 4 we prove Theorems B and C. In Section 4.1 we prove that some big sets in the dynamical plane can be extended in a holomorphic motion compatible with dynamics, in some neighborhoods of the parameter. As a consequence we obtain Theorem C. In Section 4.2 we prove that 
these holomorphic motions are close to a non-degenerate affine map near the critical value. This yields Theorem $\mathrm{B}$ about the conformality of the external maps. The non-degeneracy of this affine map is due to a transversality property proven in Appendix 2. We prove the sharpness of Theorem $\mathrm{C}$ at the end of Section 4.2.

In Section 5 we prove the HD Lemma, which is a criterion for the convergence of the Hausdorff dimension of Julia sets. Theorem A is an easy consequence of Theorem B and this lemma.

In Appendix 1 we prove two corollaries of Theorem B stated in the introduction.

In Appendix 2 we prove a transversality property. This property of semihyperbolic polynomials states that the graph of the dynamical continuation of the critical value is transversal to the diagonal. This generalizes a well known property of Misiurewicz maps; see for example [DH2]. For $d=2$ this also follows from $[\mathrm{vS}]$, which was done independently.

Acknowledgments. I am grateful to J. C. Yoccoz for reading the first version of this work, and for very useful suggestions and comments. I would also like to thank the referee for useful criticism that helped to improve the exposition.

Preliminaries. The basic facts stated here can be found in [DH2], [CG] and [CJY].

For two numbers $A$ and $B, A=\mathcal{O}(B)$ and $A \sim B$ mean $A<C B$ and $C^{-1} B<A<C B$, respectively, for some implicit constant $C$. Moreover $B_{r}(x)$ denotes the open ball of radius $r$ and center $x$.

Throughout all this work we fix $d \geq 2$ and we let $P_{c}(z)=z^{d}+c$ for $c \in \mathbb{C}$. For $c \in \mathcal{M}_{d}$, the filled-in Julia set $K_{c}$ is a compact connected set and $\overline{\mathbb{C}}-K_{c}$ is homeomorphic to $\overline{\mathbb{C}}-\overline{\mathbb{D}}$, where $\overline{\mathbb{C}}$ denotes the Riemann sphere and $\mathbb{D}$ denotes the unit disc. Moreover there is a unique conformal representation

$$
\varphi_{c}: \overline{\mathbb{C}}-K_{c} \rightarrow \overline{\mathbb{C}}-\overline{\mathbb{D}}
$$

which is tangent to the identity at infinity. This representation conjugates $P_{c}$ in $\overline{\mathbb{C}}-J_{c}$ to $z \mapsto z^{d}$ in $\overline{\mathbb{C}}-\overline{\mathbb{D}}$. For $r>1$ the set $\left\{z \mid \varphi_{c_{0}}(z)=r\right\}$ is an analytic Jordan curve called an equipotential and for $\theta \in \mathbb{R}$ the preimage of $\left\{r e^{2 \pi i \theta} \mid r>1\right\}$ under $\varphi_{c_{0}}$ is called the ray of angle $\theta$. We say that the ray of angle $\theta$ lands at $z$ if $\lim _{r \rightarrow 1} \varphi_{c_{0}}^{-1}\left(r e^{2 \pi i \theta}\right)=z$; in this case $z \in J_{c_{0}}$.

For $c \notin \mathcal{M}_{d}$, there is a map $\varphi_{c}$ defined in a neighborhood of infinity that conjugates $P_{c}$ to $z^{d}$ near infinity. Moreover we may assume that $\varphi_{c}$ is tangent to the identity at infinity. Then $\varphi_{c}$ can be extended in a canonical way to $c$. It is rather surprising that the function $\varphi_{\mathcal{M}_{d}}$, defined by $\varphi_{\mathcal{M}_{d}}(c)=\varphi_{c}(c)$ for $c \notin \mathcal{M}_{d}$, is a conformal representation of $\mathbb{C}-\mathcal{M}_{d}$ to $\mathbb{C}-\overline{\mathbb{D}}$ that is tangent to the identity at infinity; see [DH1]. 
Consider $c_{0} \in \partial \mathcal{M}_{d}$ so that $P_{c_{0}}$ is semihyperbolic. It follows from a theorem of Mañé [Ma] that all (finite) periodic points of $P_{c_{0}}$ are repelling. Moreover, the set $\omega(0)$ of accumulation points of the orbit of 0 is a hyperbolic set of $P_{c_{0}}$. Thus, by the expansive property, there is $l>1$ such that $P_{c_{0}}^{l}(0) \in$ $\omega(0)$. We suppose that $l>1$ is the least integer with this property. We usually set $z_{0}=P_{c_{0}}^{l}(0)$.

In $[\mathrm{CJY}]$ it is proved that there are constants $\varepsilon>0, C>0$ and $\theta \in(0,1)$ such that for all $x \in J_{c_{0}}$ and any connected component $B$ of $P_{c_{0}}^{-n}\left(B_{\varepsilon}(x)\right)$ for $n \geq 0$, the map

$$
P_{c_{0}}^{n}: B \rightarrow B_{\varepsilon}(x)
$$

has degree at most $d$ and $\operatorname{diam}(B)<C \theta^{n}$.

Moreover the complement of $J_{c_{0}}$ is a John domain; this means that $J_{c_{0}}$ is locally connected and there is $\delta>0$ such that if $z \in J_{c_{0}}$ and $w$ belongs to a ray landing at $z$, then $B_{\delta|z-w|}(w) \cap J_{c_{0}}=\emptyset$. In particular, by Carathéodory's theorem, the map $\varphi_{c_{0}}^{-1}$, defined in $\overline{\mathbb{C}}-\overline{\mathbb{D}}$, extends continuously to $\partial \mathbb{D}$, so every ray lands at some point in $J_{c_{0}}$.

2. Markov partitions. Fix $c_{0} \in \partial \mathcal{M}_{d}$ such that $P_{c_{0}}$ is semihyperbolic. It follows by [Ma] that $P_{c_{0}}$ is uniformly expanding in $\omega(0)$. In Section 2.1 we construct a Markov partition for $\omega(0)$ with puzzles; a puzzle is a set bounded by a finite number of (closures of) rays and an equipotential. Recall that by [CJY] all rays land at some point in $J_{c_{0}}$. Puzzles are homeomorphic to a disc.

Proposition (Markov partitions). There is a Markov partition for $\omega(0)$ with puzzles. That is, there is a finite collection of disjoint puzzles $U_{a}, a \in A$, that cover $\omega(0)$ so that $P_{c_{0}}$ is univalent in $U_{a}$ and such that if $a, b \in A$ are such that $U_{a} \cap P_{c_{0}}\left(U_{b}\right) \neq \emptyset$, then $U_{a} \subset P_{c_{0}}\left(U_{b}\right)$.

The proof of this proposition is in Section 2.1. The main reason that we need a Markov partition with puzzles, instead of any other type of set, is to have the following property needed in the proof of Proposition 4.2. If $z \notin J_{c_{0}}$ belongs to the boundary of a puzzle, then the piece of ray from $z$ to infinity is disjoint from that puzzle.

Consider the Markov partition $U_{a}, a \in A$, given by the Proposition. For $n \geq 0$, the preimages of the sets $U_{a}$ under $P_{c_{0}}^{n}$ that intersect $\omega(0)$ are called the $n$th step pieces of the Markov partition. Note that for $n \geq 1$ the collection of all the $n$th step pieces is a Markov partition; we call it a refinement of the Markov partition $U_{a}, a \in A$.

In Section 2.2 we prove that, refining the Markov partition if necessary, we have the following important property. 
Bounded Distortion Property. For any $k \geq 0$ the distortion of $P_{c_{0}}^{k}$ in each of the kth step pieces of the Markov partition is bounded by some constant $K>1$, independent of $k$.

In Section 2.2 we also consider some holomorphic motions of the Markov partition and a uniform bonded distortion property.

2.1. Construction of a Markov partition. The idea to construct the Markov partition is the following. Consider a finite set $\mathcal{P} \subset J_{c_{0}}$ of preperiodic points, which is forward invariant under $P_{c_{0}}$. There are a finite number of rays landing at a given preperiodic point; see [DH1]. Consider the collection of puzzles $U_{b}, b \in B$, determined by some equipotential and all rays that land at some point in $\mathcal{P}$. This collection of puzzles has the Markov property: if $a, b \in B$ are such that $P\left(U_{a}\right) \cap U_{b} \neq \emptyset$, then $U_{b} \subset P_{c_{0}}\left(U_{a}\right)$. The difficulty is to find such a $\mathcal{P}$ disjoint from $\omega(0)$ (so that the puzzles $U_{b}$ cover $\omega(0)$ ) and such that the puzzle containing 0 is disjoint from $\omega(0)$ (so that $P_{c_{0}}$ is univalent in every $U_{b}$ intersecting $\omega(0)$ ). Then the collection of puzzles intersecting $\omega(0)$ is the desired Markov partition.

We first reduce the situation to the non-renormalizable case; see $[\mathrm{H}]$ for references. Since all periodic points of $P_{c_{0}}$ are repelling, $P_{c_{0}}$ has $d$ fixed points, $d-1$ of them are the landing points of the $d-1$ fixed rays; we denote by $\beta$ the landing point of the ray of angle 0 . The remaining fixed point, which we denote by $\alpha$, is the landing point of $q \geq 2$ rays that are cyclically permuted by $P_{c_{0}}$.

Consider the $q$ puzzles $P_{1}, \ldots, P_{q}$ determined by the $q$ rays landing at $\alpha$ and an equipotential. Then there are two cases. Either the diameters of the successive preimages of these puzzles converge uniformly to 0 , or $P_{c_{0}}$ is renormalizable: this means that there is a pull-back $P$ of $P_{i}$, for some $1 \leq i \leq q$, that contains 0 and such that for some $n \geq 1$ we have $P \subset P^{\prime}=P_{c_{0}}^{n}(P)$ and $P_{c_{0}}^{n}: P \rightarrow P^{\prime}$ is proper of degree $d$. In this case the puzzles $P, P_{c_{0}}(P), \ldots, P_{c_{0}}^{n-1}(P)$ are pairwise disjoint and the polynomial $P_{c_{0}}^{n}$ is conjugate to some polynomial $P_{c_{1}}$ in $\bar{P}$. It follows that $P_{c_{1}}$ is also semihyperbolic. The conjugacy maps the maximal invariant set $J_{1} \subset J_{c_{0}}$ of $P_{c_{0}}^{n}$ in $\bar{P}$ to the Julia set $J_{c_{1}}$ of $P_{c_{1}}$, which is connected.

In this case it is enough to find a finite set $\mathcal{P}_{1} \subset J_{c_{1}}$ of preperiodic points of $P_{c_{1}}$, as above. In fact, if $\mathcal{P}_{1}^{\prime} \subset J_{1}$ is the set corresponding to $\mathcal{P}_{1}$, then

$$
\mathcal{P}=\mathcal{P}_{1} \cup P_{c_{0}}\left(\mathcal{P}_{1}\right) \cup \ldots \cup P_{c_{0}}^{n-1}\left(\mathcal{P}_{1}\right)
$$

is a finite set forward invariant under $P_{c_{0}}$ that has the desired properties.

As before it may happen that $P_{c_{1}}$ is renormalizable. Semihyperbolic polynomials are at most finitely renormalizable, so this process must end; see $[\mathrm{H}]$. So by the above we may assume that $P_{c_{0}}$ is not renormalizable. 
Now we consider the tree structure of $J_{c_{0}}$; see [DH1] for references. Since $J_{c_{0}}$ is a locally connected compact set with empty interior, it has a tree structure: given two different points $\delta, \gamma \in J_{c_{0}}$ there is a set $[\delta, \gamma] \subset J_{c_{0}}$, homeomorphic to a compact interval, which is the least connected subset of $J_{c_{0}}$ containing $\delta$ and $\gamma$. We write $(\gamma, \delta]=[\delta, \gamma)=[\delta, \gamma]-\{\gamma\}$ and $(\gamma, \delta)=$ $[\gamma, \delta)-\{\gamma\}$. Such sets are called arcs. Moreover for every $\delta_{0}, \delta_{1}, \delta_{2} \in J_{c_{0}}$ there is $\gamma \in J_{c_{0}}$ so that $\left[\delta_{0}, \delta_{1}\right] \cap\left[\delta_{0}, \delta_{2}\right]=\left[\delta_{0}, \gamma\right]$.

It is easy to see that $\alpha \in\left[\beta, c_{0}\right]$ and that if $P_{c_{0}}$ is not injective in an arc, then the critical point, 0 , belongs to it. If $w \in J_{c_{0}}$ is such that there are $\gamma$ and $\delta$ in $J_{c_{0}}$ such that $w \in(\gamma, \delta)$, then $w$ is the landing point of at least two rays. The following property is well known.

Lemma 2.1. Let $\gamma \in J_{c_{0}}$ be the landing point of at least two rays. Then there is $k$ such that $P_{c_{0}}^{k}(\gamma) \in\left[\alpha, c_{0}\right]$.

Proof. Let $\theta^{+}$and $\theta^{-}$be different angles of rays landing at $\gamma$. By iterating if necessary, we may assume that there is $i \in\{1, \ldots, d-1\}$ such that $\theta^{+}$and $\theta^{-}$lay in different connected components of $\mathbb{T}-\{i / d, 0\}$, where $\mathbb{T}=\mathbb{R} / \mathbb{Z}$; equivalently there is a preimage $\beta^{\prime}$ of $\beta$ so that $\gamma \in\left(\beta, \beta^{\prime}\right)$. Note that $0 \in\left(\beta, \beta^{\prime}\right)$ and therefore

$$
P_{c_{0}}((\beta, 0))=P_{c_{0}}\left(\left(\beta^{\prime}, 0\right)\right)=\left(\beta, c_{0}\right) .
$$

Since $\alpha \in\left(\beta, c_{0}\right)$ there is a preimage $\alpha^{\prime}$ of $\alpha$ in $(\beta, \alpha)$, so that

$$
P_{c_{0}}((\alpha, 0))=P_{c_{0}}\left(\left(\alpha^{\prime}, 0\right)\right)=\left(\alpha, c_{0}\right),
$$

and $P_{c_{0}}\left(\left(\beta, \alpha^{\prime}\right)\right)=(\beta, \alpha)$. So for every $\delta \in\left(\beta, c_{0}\right)$ there is $n \geq 0$ so that $P_{c_{0}}^{n}(\delta) \in\left[\alpha, c_{0}\right]$. Since $P_{c_{0}}(\gamma) \in\left(\beta, c_{0}\right]$, the lemma follows.

Lemma 2.2. Suppose that $P_{c_{0}}$ is not renormalizable. Then:

(i) The preimages of $\alpha$ and the preimages of 0 are dense in $(0, \alpha)$.

(ii) For any $\gamma \in J_{c_{0}}-\{\alpha\}$ there are $\delta \in(\alpha, \gamma)$ and $k \geq 1$ such that $P_{c_{0}}^{k}$ is univalent in $(\alpha, \delta)$ and $P_{c_{0}}^{k}((\alpha, \delta))=(\alpha, 0)$.

(iii) The periodic points of $P_{c_{0}}$ are dense in $(\alpha, 0)$.

(iv) If $c_{0}$ is not preperiodic, there is $k \geq 0$ such that for every $\gamma \in$ $J_{c_{0}}-\left\{P_{c_{0}}^{k}(0)\right\}$ there is a periodic point of $P_{c_{0}}$ in $\left(P_{c_{0}}^{k}(0), \gamma\right)$ that is not in $\omega(0)$.

Proof. (i) Note that the boundary of a preimage of a puzzle $P_{i}$ intersects $J_{c_{0}}$ at preimages of $\alpha$. Since the closures of the $n$th preimages of the $P_{i}$, for $1 \leq i \leq q$, cover $J_{c_{0}}$ and have small diameter, it follows that for every subarc $I \subset(0, \alpha)$ there is a preimage $P$ of some $P_{i}$ intersecting $I$ with diameter much smaller than that of $I$. So $\partial P \cap I \subset \partial P \cap J_{c_{0}}$ is not empty and therefore it contains a preimage of $\alpha$. To prove that the preimages of 0 are dense in $I$, 
note that by the above, $I$ contains at least two preimages of $\alpha$. Therefore there is $k \geq 1$ so that $P_{c_{0}}^{k}$ is not injective in $I$, thus $I$ contains a preimage of 0 .

(ii) Iterating at most $q-1$ times we may suppose that $\gamma$ belongs to the same connected component of $J_{c_{0}}-\{\alpha\}$ that contains 0 ; so $(\alpha, \gamma] \cap(\alpha, 0]$ is of the form $(a, \tilde{\gamma}]$ and we may suppose that $\gamma$ belongs to $(\alpha, 0]$. By $(\mathrm{i}),(\alpha, \gamma)$ contains a $k$ th preimage $\delta$ of 0 ; we suppose that $\delta$ minimizes $k$, so that $P_{c_{0}}^{k}$ is univalent in $(\alpha, \delta)$ and $P_{c_{0}}^{k}((\alpha, \delta))=(\alpha, 0)$.

(iii) Consider a subarc $I$ of $(\alpha, 0)$. By (i) there is a preimage $\gamma$ of $\alpha$ in $I$; let $k$ be such that $P_{c_{0}}^{k}(\gamma)=\alpha$ and $\gamma^{\prime} \in I-\{\gamma\}$ so that $P_{c_{0}}^{k}$ is univalent in $\left(\gamma, \gamma^{\prime}\right)$. By (ii), taking $\gamma^{\prime}$ closer to $\gamma$ if necessary, we may assume that there is $l$ so that $P_{c_{0}}^{l}$ is univalent in $\left(P_{c_{0}}^{k}(\gamma), P_{c_{0}}^{k}\left(\gamma^{\prime}\right)\right)$ and $P_{c_{0}}^{k+l}\left(\left(\gamma, \gamma^{\prime}\right)\right)=(\alpha, 0)$. Thus there is a periodic point of $P_{c_{0}}$ in $I$.

(iv) If there are at least three rays landing at $c_{0}$, then $c_{0}$ is preperiodic; see $[\mathrm{Th}]$ and $[\mathrm{K}]$. So there is nothing to prove in this case. So we assume that there are at most two rays landing at $c_{0}$.

If there is only one ray landing at $c_{0}$ then for every $\delta \in J_{c_{0}}-\left\{c_{0}\right\}$, the arc $\left(c_{0}, \delta\right)$ contains a subarc of $\left(\alpha, c_{0}\right)$. By (iii) the periodic points of $P_{c_{0}}$ are dense in $\left(\alpha, c_{0}\right)$ so the assertion follows with $k=1$, by considering that $c_{0}$ is not in $\omega(0)$.

If there are exactly two rays landing at $c_{0}$ and $c_{0}$ is not eventually mapped to $\alpha$, then by Lemma 2.1 there is $l \geq 0$ so that $P_{c_{0}}^{l}\left(c_{0}\right) \in\left(\alpha, c_{0}\right)$. Then for every $\gamma \in J_{c_{0}}-\left\{P_{c_{0}}^{l}(0)\right\}$, the arc $\left(P_{c_{0}}^{l}(0), \gamma\right)$ contains a subarc of $(\alpha, 0)$. By (i) the preimages of 0 are dense in $(\alpha, 0)$ so $\omega(0)$ is nowhere dense in $(\alpha, 0)$. On the other hand, by (iii), the periodic points of $P_{c_{0}}$ are dense in $(\alpha, 0)$ so the assertion follows with $k=l+1 \geq 1$.

Proof of the Proposition. As already mentioned we may suppose that $P_{c_{0}}$ is not renormalizable, so the previous lemma applies. If $c_{0}$ is preperiodic and not eventually mapped to $\alpha$ we can construct a Markov partition for the finite set $\omega(0)$ with preimages of the puzzles constructed with $\alpha$. If $c_{0}$ is eventually mapped to $\alpha$ then clearly in the previous lemma property (iii) implies (iv). So we can assume that $P_{c_{0}}$ satisfies (iv) of the previous lemma.

Let $k \geq 1$ be as in (iv) of the previous lemma and let $\delta>0$ be such that $\left|P_{c_{0}}^{k}(0)\right|>\delta$. So by the previous lemma, for every $y \in \partial B_{\delta}\left(P_{c_{0}}^{k}(0)\right) \cap J_{c_{0}}$ there is a periodic point $p(y) \in\left(y, P_{c_{0}}^{k}(0)\right)$ that is not in $\omega(0)$. Moreover we may suppose that $p(y) \in B_{\delta}\left(P_{c_{0}}^{k}(0)\right)$.

Since $p(y)$ belongs to the arc $\left(y, P_{c_{0}}^{k}(0)\right)$ there are at least two rays that land at $p(y)$. These rays divide $\mathbb{C}$ in at least two parts; let $U(y)$ be the one containing $y$. Since $\partial B_{\delta}\left(P_{c_{0}}^{k}(0)\right) \cap J_{c_{0}}$ is a compact set, we may choose points $y_{1}, \ldots, y_{n}$ in this set so that the $U\left(y_{i}\right)$ for $1 \leq i \leq n$ cover $\partial B_{\delta}\left(P_{c_{0}}^{k}(0)\right) \cap J_{c_{0}}$. 
Consider the puzzle $P$ containing $P_{c_{0}}^{k}(0)$ determined by all rays that land at the points $p\left(y_{1}\right), \ldots, p\left(y_{n}\right)$ and some equipotential. Choosing the equipotential with sufficiently small potential, we may assume that the puzzle $P$ is contained in $B_{\delta}\left(P_{c_{0}}^{k}(0)\right)$. So $\bar{P}$ does not contain 0 .

Let $\mathcal{P}$ be the set of all points in the forward orbit of points in $P_{c_{0}}^{-k}\left(p\left(y_{i}\right)\right)$ for $1 \leq i \leq n$. So $\mathcal{P}$ is a finite set of preperiodic points that is forward invariant under $P_{c_{0}}$ and is disjoint from $\omega(0)$ by construction. Consider the collection $U_{b}, b \in B$, of the puzzles determined by the rays that land at points in $\mathcal{P}$ and some equipotential. By construction the puzzle containing the critical point is disjoint from $\omega(0)$. Then, as remarked above, the collection $U_{a}, a \in A \subset B$, of the puzzles that intersect $\omega(0)$ forms a Markov partition for $\omega(0)$.

2.2. Bounded distortion property and holomorphic motions. Consider the Markov partition $U_{a}, a \in A$, for $\omega(0)$ given by the Proposition. Refining the Markov partition if necessary we prove that it has the property stated below. As in immediate consequence, together with the Koebe Distortion Theorem, we obtain the Bounded Distortion Property.

Univalent Extension Property. Let $W$ be an $n$th step piece of the Markov partition $U_{a}, a \in A$. Then the inverse of

$$
P_{c_{0}}^{n}: W \rightarrow U_{a}=P_{c_{0}}^{n}(W)
$$

extends in a univalent way to a neighborhood of $\bar{U}_{a}$, only depending on a $\in A$.

Proof. We will prove that there is $m \geq 1$ such that all the $m$ th step pieces of the Markov partition are compactly contained in some $U_{a}$. Then the Markov partition formed by the $m$ th step pieces will be the desired Markov partition. Thus it is enough to prove that the diameters of the $m$ th step pieces of the Markov partition converge uniformly to zero as $m \rightarrow \infty$.

Let $\varepsilon>0, C>0$ and $\theta \in(0,1)$ be as in [CJY]; see Preliminaries. Let $N \geq 1$ be such that we can partition each $U_{a}, a \in A$, in at most $N$ connected sets of diameter less than $\varepsilon>0$. Let $W$ be an $m$ th step piece of the Markov partition, so that $P_{c_{0}}^{m}$ is univalent in $W$. Then by [CJY] it follows that $\operatorname{diam}(W) \leq N C \theta^{m}$.

Since $P_{c_{0}}$ is uniformly expanding in $\omega(0)$ there is a holomorphic motion $j: B_{\delta}\left(c_{0}\right) \times \omega(0) \rightarrow \mathbb{C}$, for some $\delta>0$, which is compatible with dynamics; see [Sh]. This means that for each $c \in B_{\delta}\left(c_{0}\right)$ the map $j_{c}: \omega(0) \rightarrow \mathbb{C}$ is injective and for each $z \in \omega(0)$ the function $c \mapsto j_{c}(z)$ is holomorphic. Being compatible with dynamics means that for every $c \in B_{\delta}\left(c_{0}\right)$ the map $j_{c}$ conjugates $P_{c_{0}}$ on $\omega(0)$ to $P_{c}$ on $j_{c}(\omega(0))$.

Recall that $l>1$ is the least integer such that $P_{c_{0}}^{l}(0) \in \omega(0)$; see Preliminaries. Reducing $\delta>0$ if necessary we extend the holomorphic motion 
$j$ to $c_{0}$; thus the function $z(c)=j_{c}\left(c_{0}\right)$ is the dynamical continuation of the critical value $c_{0}$. This holomorphic function will be important for Theorem B. In fact the similarity factor is $\lambda=1-z^{\prime}\left(c_{0}\right)$ and the Transversality property proven in Appendix 2 is that $\lambda=1-z^{\prime}\left(c_{0}\right) \neq 0$. Note that the function $z$ is defined in $B_{\delta}\left(c_{0}\right)$, it satisfies $z\left(c_{0}\right)=c_{0}$ and by definition $P_{c}^{l-1}(z(c))=j_{c}\left(P_{c_{0}}^{l-1}\left(c_{0}\right)\right)$.

Proposition 2.3. Consider a Markov partition $U_{a}, a \in A$, of $\omega(0)$ as in Section 2.1. Then there is $\delta>0$ and a holomorphic motion $j: B_{\delta}\left(c_{0}\right) \times$ $\bigcup_{a \in A} U_{a} \rightarrow \mathbb{C}$ compatible with dynamics. Moreover there is $R>0$ such that $j\left(B_{\delta}\left(c_{0}\right) \times \bigcup_{a \in A} U_{a}\right) \subset B_{R}(0)$.

Proof. Let $\mathcal{P} \subset J_{c_{0}}$ be the finite set of preperiodic points used in the construction of the Markov partition $U_{a}, a \in A$. By construction $\mathcal{P}$ is forward invariant and does not contain 0 . By reducing $\delta>0$ we may suppose that $j$ is also defined in $\mathcal{P}$. Thus for any $z \in \mathcal{P}$ and any $c \in B_{\delta}\left(c_{0}\right), j_{c}(z)$ is a preperiodic point of $P_{c}$. Moreover the rays of $P_{c}$ landing at $j_{c}(z)$ have the same angles as those of $P_{c_{0}}$ landing at $z$ and we may extend the holomorphic motion $j$ to the set of rays landing at points of $\mathcal{P}$. Extending $j$ to the equipotential used in the construction of the Markov partition (reducing $\delta>0$ if necessary) we may suppose that $j$ is defined in $\bigcup_{a \in A} \partial U_{a}$. By [St] we may extend $j$ to a fundamental domain of the Markov partition, and then to $\bigcup_{a \in A} U_{a}$ by dynamics.

Since for all $c \in B_{\delta}\left(c_{0}\right), j_{c}\left(\bigcup_{a \in A} U_{a}\right)$ is contained in the set bounded by an equipotential with potential independent of $c$, it follows that there is $R>0$ such that $j\left(B_{\delta}\left(c_{0}\right) \times \bigcup_{a \in A} U_{a}\right) \subset B_{R}(0)$.

We end this section with the following lemma, which is independent of dynamics and is used in the proof of Lemma 4.3.

Lemma 2.4. Let $j: \mathbb{D} \times X \rightarrow \mathbb{C}$ be a holomorphic motion such that $j(\mathbb{D} \times X) \subset B_{R}(0)$ for some $R>0$. If $x, y \in X$ and $z \in \mathbb{D}$ are such that $|z|\left(\ln \left(\frac{1}{2 R}|x-y|\right)\right)^{-1} \leq \frac{1}{4}$, then

$$
\left|j_{z}(x)-j_{z}(y)-(x-y)\right| \leq 4|z| \cdot|x-y|\left(\ln \left(\frac{1}{2 R}|x-y|\right)\right)^{-1} .
$$

Proof. Fix $y \in X$ and consider the holomorphic motion $i: \mathbb{D} \times X \rightarrow \mathbb{D}$ defined by $i(x)=(2 R)^{-1}\left(j_{z}(x)-j_{z}(y)\right)$, so for $x \neq y, i_{z}(x) \in \mathbb{D}-\{0\}$. Moreover fix $x \in X-\{y\}$ and put $x_{z}=i_{z}(x)$ for $z \in \mathbb{D}$. The map $w \mapsto$ $x_{0} e^{\frac{-2 w}{1+w} \ln \left|x_{0}\right|}$ is a local isometry between $\mathbb{D}$ and $\mathbb{D}-\{0\}$ which maps 0 to $x_{0}=x$. Therefore by Schwarz' Lemma,

$$
x_{z} \in\left\{w \in \mathbb{D}-\{0\} \mid \varrho_{\mathbb{D}-\{0\}}\left(w, x_{0}\right) \leq \varrho_{\mathbb{D}}(0, z)\right\}=\left\{x_{0} e^{\frac{-2 w}{1+w} \ln \left|x_{0}\right|}|| w|\leq| z \mid\right\},
$$

where $\varrho_{\mathbb{D}-\{0\}}$ and $\varrho_{\mathbb{D}}$ denote the hyperbolic distances in $\mathbb{D}-\{0\}$ and in $\mathbb{D}$ 
respectively. The hypothesis $|z| \ln \left(1 /\left|x_{0}\right|\right) \leq 1 / 4$ implies that

$$
\left|e^{\frac{2|z|}{1+|z|} \ln \frac{1}{\left|x_{0}\right|}}-1\right| \leq 4|z| \ln \frac{1}{\left|x_{0}\right|}
$$

SO

$$
\begin{aligned}
\left|x_{z}-x_{0}\right| & \leq \sup _{|w|<|z|}\left|x_{0} e^{\frac{-2 w}{1+w} \ln \left|x_{0}\right|}-x_{0}\right|=\left|x_{0}\right| \cdot\left|e^{\frac{2|z|}{1+|z|} \ln \frac{1}{\left|x_{0}\right|}}-1\right| \\
& \leq 4|z| \cdot\left|x_{0}\right| \ln \frac{1}{\left|x_{0}\right|}
\end{aligned}
$$

3. Expansion and backward stability. Fix $c_{0} \in \partial \mathcal{M}_{d}$ such that $P_{c_{0}}$ is semihyperbolic. We begin this section by proving that $P_{c_{0}}$ has the Almost Uniform Expansion property stated below. As a consequence we prove the Main Lemma in Section 3.1, about backward stability of semihyperbolic polynomials.

Consider the constants $\varepsilon>0, C>0$ and $\theta \in(0,1)$ as in [CJY]; see Preliminaries.

Almost Uniform Expansion. There is a constant $A>0$ such that given $z \in \mathbb{C}$ and $k \geq 0$, if $0 \leq j<k$ is such that $\left|P_{c_{0}}^{j}(z)\right| \leq\left|P_{c_{0}}^{i}(z)\right|$ for all $0 \leq i<k$, then

$$
\left|\left(P_{c_{0}}^{j}\right)^{\prime}(z)\right| \geq A \theta^{-j} \quad \text { and } \quad\left|\left(P_{c_{0}}^{k-j-1}\right)^{\prime}\left(P_{c_{0}}^{j+1}(z)\right)\right| \geq A \theta^{-(k-j-1)} .
$$

This property is equivalent to the Collet-Eckmann condition, which requires a positive Lyapunov exponent at the critical value; see [R-L3]. In the presence of more than one critical point this is no longer true.

The following is a distortion lemma for ramified maps, which is independent of dynamics.

Lemma 3.1. Let $N \geq 1$ and $\gamma \in(0,1)$ be given. Then there exists $\kappa=$ $\kappa(\gamma, N) \in(0,1)$ such that for any open and simply connected bounded set $U \subset \mathbb{C}$ and any ramified covering $R: U \rightarrow \mathbb{D}$ of degree $N$, we have

$$
\operatorname{diam}\left(U^{\prime}\right) \leq \gamma \operatorname{diam}(U)
$$

where $U^{\prime}$ is any connected component of $R^{-1}\left(B_{\kappa}(0)\right)$.

Proof. It is enough to prove that the hyperbolic diameter of $U^{\prime}$ in $U$ goes to zero as $\kappa \rightarrow 0$. So suppose that $U=\mathbb{D}$ and $R(0)=0$; so there are $a_{1}, \ldots, a_{N-1} \in \mathbb{D}$ and $\lambda \in \partial \mathbb{D}$ such that

$$
R(z)=\lambda z \prod_{i=1}^{N-1} \frac{z-a_{i}}{1-\bar{a}_{i} z} .
$$


Note that for any $\nu \in(0,1)$ we may choose $\frac{1}{2} \nu<\xi<\nu$ such that for any $w$ satisfying $|w|=\xi$ we have $\left|w-a_{i}\right| \geq \nu /(4 N)$ for $1 \leq i<N$. So

$$
|R(w)|=\left|\lambda \prod_{i=1}^{N-1} \frac{w-a_{i}}{1-\bar{a}_{i} w}\right| \geq\left(\frac{\nu}{8 N}\right)^{N-1} .
$$

Thus for $\kappa(N, \gamma)=(\nu /(8 N))^{N-1}$ we have $U^{\prime} \subset B_{\nu}(0)$. So the hyperbolic diameter of $U^{\prime}$ goes to 0 as $\kappa \rightarrow 0$.

Let $\kappa \in(0,1)$ be as in Lemma 3.1 for $N=d$ and $\gamma=C^{-1 \varepsilon / 2}$, so that by [CJY] for any $z \in B_{\kappa \varepsilon / 2}\left(J_{c_{0}}\right)$, any $n \geq 0$ and any connected component $W$ of $P_{c_{0}}^{-n}\left(B_{\kappa \varepsilon / 2}(z)\right)$, we have $\operatorname{diam}(W)<\varepsilon / 2$.

LEMMA 3.2. Let $\kappa \in(0,1)$ be as above and let $\delta>0$ be such that for all $z$ and $k \geq 1$ such that $P_{c_{0}}^{k}(z) \in B_{\delta}\left(J_{c_{0}}\right)$ we have $P_{c_{0}}^{i}(z) \in B_{\kappa \varepsilon / 2}\left(J_{c_{0}}\right)$ for $0 \leq i \leq k+1$. Then there is a constant $A_{0}>0$ such that for all $z$ and $k$ such that $P_{c_{0}}^{k}(z) \in B_{\varepsilon / 2}(0)$ or $P_{c_{0}}^{k}(z) \notin B_{\delta}\left(J_{c_{0}}\right)$ we have

$$
\left|\left(P_{c_{0}}^{k}\right)^{\prime}(z)\right| \geq A_{0} \max \left(\theta^{-k},|z|^{-1}\right) \text {. }
$$

Proof. If $P_{c_{0}}^{k}(z) \in B_{\varepsilon / 2}(0)$ then the pull-back of $B_{\varepsilon}(0)$ by $P_{c_{0}}^{k}$ to $z$ is univalent, so by the Koebe $\frac{1}{4}$ Theorem $\left|\left(P_{c_{0}}^{k}\right)^{\prime}(z)\right| \geq \frac{1}{4}(\varepsilon / 2)|z|^{-1}$. By [CJY] and Schwarz' Lemma we have $\left|\left(P_{c_{0}}^{k}\right)^{\prime}(z)\right| \geq(\varepsilon / 2) C^{-1} \theta^{-k}$, so the lemma follows in this case with constant $A_{1}=(\varepsilon / 2) \min \left(1 / 4, C^{-1}\right)$.

Suppose that $P_{c_{0}}^{k}(z) \notin B_{\delta}\left(J_{c_{0}}\right)$, so we may assume that $k \geq 1$. Note that there is a constant $A_{2}>0$ such that for all $w \notin B_{\delta}\left(J_{c_{0}}\right)$ and all $n \geq 0$ we have $\left|\left(P_{c_{0}}^{n}\right)^{\prime}(z)\right| \geq A_{2} \theta^{-n}$. Thus we may assume that $P_{c_{0}}^{k-1}(z) \in B_{\delta}\left(J_{c_{0}}\right)$, so $P_{c_{0}}^{i}(z) \in B_{\kappa \varepsilon / 2}\left(J_{c_{0}}\right)$ for $0 \leq i \leq k$.

If $P_{c_{0}}^{m}(z) \notin B_{\varepsilon / 2}(0)$ for $0 \leq m<k$, then the pull-back of $B_{\kappa \varepsilon / 2}\left(P_{c_{0}}^{k}(z)\right)$ to $z$ is univalent and the lemma follows as before in this case with constant $\kappa A_{1}$.

Otherwise let $0 \leq m<k$ be the greatest integer such that $P_{c_{0}}^{m}(z) \in$ $B_{\varepsilon / 2}(0)$. As before the pull-back of $B_{\kappa \varepsilon / 2}\left(P_{c_{0}}^{k}(z)\right)$ by $P_{c_{0}}^{k-m}$ to $P_{c_{0}}^{m}(z)$ is univalent so $\left|\left(P_{c_{0}}^{k-m}\right)^{\prime}(z)\right| \geq \kappa A_{1} \max \left(\theta^{-(k-m)},\left|P_{c_{0}}^{m}(z)\right|^{-1}\right)$; thus the assertion holds if $m=0$. If $m>0$ we have $\left|\left(P_{c_{0}}^{m}\right)^{\prime}(z)\right| \geq \kappa A_{1} \max \left(\theta^{-m},|z|^{-1}\right)$, so the lemma follows.

Proof of the Almost Uniform Expansion property. Let $\kappa \in(0,1)$ be as in Lemma 3.1 for $N=d$ and $\gamma=C^{-1} \varepsilon / 2$ as before and let $\delta>0$ be as in Lemma 3.2. As in the proof of Lemma 3.2 we may assume that $P_{c_{0}}^{k}(z)$ belongs to $B_{\delta}\left(J_{c_{0}}\right)$ so that $P_{c_{0}}^{i}(z) \in B_{\kappa \varepsilon / 2}\left(J_{c_{0}}\right)$ for $0 \leq i \leq k$. Consider the pull-back $B_{i}$ of $B_{k}=B_{\kappa \varepsilon / 2}\left(P_{c_{0}}^{k}(z)\right)$ by $P_{c_{0}}^{k-i}$ to $P_{c_{0}}^{i}(z)$; so by definition of $\kappa$ we have $\operatorname{diam}\left(B_{i}\right)<\varepsilon / 2$.

If $\left|P_{c_{0}}^{j}(z)\right| \geq \varepsilon / 2$ then $P_{c_{0}}^{k-j}$ is univalent in $B_{j}$ so $\left|\left(P_{c_{0}}^{k-j}\right)^{\prime}(z)\right|^{-1} \geq$ $(\varepsilon / 2) C^{-1} \theta^{-(k-\bar{j})}$ by Schwarz' Lemma. In a similar way the pull-back of 
$B_{\kappa \varepsilon / 2}\left(P_{c_{0}}^{j}(z)\right)$ by $P_{c_{0}}^{j}$ to $z$ is univalent and $\left|\left(P_{c_{0}}^{j}\right)^{\prime}(z)\right| \geq A_{3} \theta^{-j}$, where $A_{3}=$ $\kappa(\varepsilon / 2) C^{-1}$.

If $\left|P_{c_{0}}^{j}(z)\right|<\varepsilon / 2$ we see by Lemma 3.2 that $\left|\left(P_{c_{0}}^{j}\right)^{\prime}(z)\right| \geq A_{0} \theta^{-j}$. If $P_{c_{0}}^{k-j}$ is univalent in $B_{j}$ then $\left|\left(P_{c_{0}}^{k-j}\right)^{\prime}(z)\right| \geq A_{3} \theta^{-(k-j)}$ and we are done. Otherwise there is a unique $j \leq i<k$ so that $0 \in B_{i}$. In this case $B_{i} \subset B_{\varepsilon / 2}(0)$, so by Lemma $3.2,\left|\left(P_{c_{0}}^{j-i}\right)^{\prime}(z)\right| \geq A_{0} \theta^{-(j-i)}$ and on the other hand $P_{c_{0}}^{k-i-1}$ is univalent in $B_{i+1}$ so $\left|\left(P_{c_{0}}^{k-i-1}\right)^{\prime}\left(P_{c_{0}}^{i+1}(z)\right)\right| \geq A_{3} \theta^{-(k-i-1)}$. Considering that by hypothesis $\left|P_{c_{0}}^{\prime}\left(P_{c_{0}}^{j}(z)\right)\right|^{-1} \geq\left|P_{c_{0}}^{\prime}\left(P_{c_{0}}^{i-1}(z)\right)\right|^{-1}$, it follows that

$$
\left|P_{c_{0}}^{i-j-1}\left(P_{c_{0}}^{j+1}(z)\right)\right| \geq\left|P_{c_{0}}^{i-j-1}\left(P_{c_{0}}^{j}(z)\right)\right| \geq A_{3} \theta^{-(i-j-1)} .
$$

3.1. Backward stability and shadowing. In this section we prove the following lemma.

Main Lemma. Let $V$ be the neighborhood of $J_{c_{0}}$ bounded by the equipotential with potential 1 . Then there is a constant $M>0$, only depending on $P_{c_{0}}$, such that for $\varrho>0$ small there is a finite collection $\left\{D_{i}\right\}$ of open sets with the following properties:

- $\operatorname{diam}\left(D_{i}\right) \leq M \varrho^{1 / d}$.

- For all $z \in V$ there is $D_{i}$ such that $B_{\varrho}(z) \subset D_{i}$.

- For all $D_{i}$ and any connected component $W$ of $P_{c_{0}}^{-1}\left(B_{\varrho}\left(D_{i}\right)\right)$ there is $D_{j}$ such that $W \subset D_{j}$.

The proof of the Main Lemma is at the end of this section and is based on Lemmas 3.3 and 3.4 below. Note that this lemma implies that for $\varrho>0$ small any $\varrho$ backward pseudo-orbit in $V$ is $M \varrho^{1 / d}$-shadowed by a backward orbit of $P_{c_{0}}$.

Let us introduce some notation for the next lemmas. Fix $\alpha \in(1 / d-$ $\left.1 / d^{2}, 1 / d\right)$ only depending on $d$. For given $R>0$ and $\varrho>0$ small put $\eta=1+M_{1} R \varrho^{1 / d-\alpha}$, for some $M_{1}>0$ to be chosen in Lemma 3.4. Let $V$ be as in the Main Lemma and put $V^{\prime}=P_{c_{0}}^{-1}(V) \subset V$. If $\zeta$ is a preimage of 0 or $\zeta \in V-J_{c_{0}}$ let $r_{\zeta}$ be defined by

$$
r_{\zeta}= \begin{cases}R \varrho^{1 / d} & \text { if }|\zeta|<\varrho^{\alpha}, \\ R \varrho & \text { if } \zeta \in V-V^{\prime} \\ r_{\zeta}=\eta\left|P_{c_{0}}^{\prime}(\zeta)\right|^{-1}\left(r_{P_{c_{0}}(\zeta)}+4 \varrho\right) & \text { otherwise. }\end{cases}
$$

Lemma 3.3. There is $M_{2}>0$ only depending on $P_{c_{0}}$ such that if $\varrho$ is small enough then for every preimage $\zeta$ of 0 and for $\zeta \in V-J_{c_{0}}$, we have $r_{\zeta} \leq M_{2} R \varrho^{1 / d}$.

Proof. Let $\zeta$ be a preimage of 0 or let $\zeta \in V-J_{c_{0}}$. So there is $n \geq 0$ such that $P_{c_{0}}^{n}(\zeta) \in B_{\varrho^{\alpha}}(0)$, resp. $P_{c_{0}}^{n}(\zeta) \in V-V^{\prime}$; let $n$ be minimal with this 
property. Then we have the following recursive formula for $r_{\zeta}$ :

$$
r_{\zeta}=\eta^{n}\left|\left(P_{c_{0}}^{n}\right)^{\prime}(\zeta)\right|^{-1} r_{P_{c_{0}}^{n}(\zeta)}+4 \varrho \sum_{m=1}^{n} \eta^{m}\left|\left(P_{c_{0}}^{m}\right)^{\prime}(\zeta)\right|^{-1},
$$

where $r_{P_{c_{0}}^{n}(z)}=R \varrho$ or $R \varrho^{1 / d}$. By Lemma $3.2,\left|\left(P_{c_{0}}^{n}\right)^{\prime}(\zeta)\right|^{-1} \geq A_{0} \theta^{-n}$. So if $\varrho>0$ is small enough so that $\eta \theta<1$ we have

$$
\eta^{n}\left|\left(P_{c_{0}}^{n}\right)^{\prime}(\zeta)\right|^{-1} \leq A_{0}^{-1}(\eta \theta)^{n} \leq A_{0}^{-1} .
$$

By the Almost Uniform Expansion property, for all $m \leq n$ we have $\left|\left(P_{c_{0}}^{m}\right)^{\prime}(\zeta)\right|$ $\geq A^{2} \theta^{-(m-1)} d\left(\varrho^{\alpha}\right)^{d-1}$. Therefore there is a constant $M_{3}>0$ only depending on $P_{c_{0}}$ such that for $\varrho$ small,

$$
r_{\zeta} \leq M_{3}\left(R \varrho^{1 / d}+\varrho^{1-(d-1) \alpha}\right) \leq 2 M_{3} R \varrho^{1 / d},
$$

the last considering that by definition $\alpha<1 / d$.

Lemma 3.4. For appropriate choices of $M_{1}>0$ and $R>0$, only depending on $P_{c_{0}}$, if $\varrho>0$ is small enough, then for any preimage $\zeta \neq 0$ of 0 and for any $\zeta \in V^{\prime}-J_{c_{0}}$, if $W$ is the connected component of

$$
P_{c_{0}}^{-1}\left(B_{r_{P_{0}(\zeta)}+4 \varrho}\left(P_{c_{0}}(\zeta)\right)\right)
$$

containing $\zeta$, then $W \subset B_{r_{\zeta}}(\zeta)$.

Proof. 1. Suppose $|\zeta| \geq \varrho^{\alpha}$ so by definition $r_{\zeta}=\eta\left|P_{c_{0}}^{\prime}(\zeta)\right|^{-1}\left(r_{P_{c_{0}}(\zeta)}+4 \varrho\right)$. Note that $P_{c_{0}}$ is univalent in $B_{\mu_{d} \varrho^{\alpha}}(\zeta)$ where $\mu_{d}>0$ is a constant that only depends in $d$ (the degree of $P_{c_{0}}$ ). By the previous lemma $r_{\zeta} \leq M_{2} R \varrho^{1 / d}$, so the distortion of $P_{c_{0}}$ in $B_{r_{\zeta}}(\zeta)$ is bounded by $1+K M_{2} R \varrho^{1 / d-\alpha}$ for some constant $K>0$ given by the Koebe Distortion Theorem. Since $M_{2}$ only depends on $P_{c_{0}}$, we may choose a priori $M_{1}=K M_{2}$ so that the distortion of $P_{c_{0}}$ in $B_{r_{\zeta}}(\zeta)$ is bounded by $\eta=1+M_{1} R \varrho^{1 / d-\alpha}$. Therefore $P_{c_{0}}\left(B_{r_{\zeta}}(\zeta)\right)$ contains the ball of radius $\eta^{-1}\left|P_{c_{0}}^{\prime}(\zeta)\right| r_{\zeta}=r_{P_{c_{0}}}+4 \varrho$ centered at $P_{c_{0}}(\zeta)$.

2. Now suppose that $|\zeta|<\varrho^{\alpha}$ and put $\zeta_{1}=P_{c_{0}}(\zeta)$. We will prove that there is a constant $K_{1}>0$ only depending on $P_{c_{0}}$, and not on $R$, so that if $\varrho$ is small enough then $r_{\zeta_{1}} \leq K_{1} \varrho$. Let $n>1$ be the first integer such that $\left|P_{c_{0}}^{n}\left(\zeta_{1}\right)\right|<\varrho^{\alpha}$, resp. $P_{c_{0}}^{n}\left(\zeta_{1}\right) \in V^{\prime}-V$. Thus $\left|P_{c_{0}}^{\prime}(\zeta)\right| \leq\left|P_{c_{0}}^{\prime}\left(P_{c_{0}}^{m}\left(\zeta_{1}\right)\right)\right|$ for $0 \leq m<n$, and by the Almost Uniform Expansion property applied to $z=\zeta$, we have $\left|\left(P_{c_{0}}^{m}\right)^{\prime}\left(\zeta_{1}\right)\right|^{-1} \leq A^{-1} \theta^{m}$, so

$$
\sum_{m=1}^{n-1} \eta^{m}\left|\left(P_{c_{0}}^{m}\right)^{\prime}\left(\zeta_{1}\right)\right|^{-1} \leq K_{2}=A^{-1} \frac{\eta \theta}{1-\theta \eta} .
$$

On the other hand, by Lemma 3.2 we have

$$
\begin{gathered}
d|\zeta|^{d-1}\left|\left(P_{c_{0}}^{n}\right)^{\prime}\left(\zeta_{1}\right)\right|=\left|\left(P_{c_{0}}^{n+1}\right)^{\prime}(\zeta)\right| \geq A_{0} \max \left(\theta^{-n},|\zeta|^{-1}\right), \quad \text { so } \\
\left|\left(P_{c_{0}}^{n}\right)^{\prime}\left(\zeta_{1}\right)\right| \geq A_{0} d^{-1}|\zeta|^{-d} \geq A_{0} d^{-1} \varrho^{-d \alpha}
\end{gathered}
$$


and we may suppose $\varrho>0$ small enough so that $\left|\left(P_{c_{0}}^{n}\right)^{\prime}\left(\zeta_{1}\right)\right| \geq \theta^{-n}$. Thus $\eta^{n}=\theta^{-n \beta} \leq\left|\left(P_{c_{0}}^{n}\right)^{\prime}\left(\zeta_{1}\right)\right|^{\beta}$, where $\beta=-\ln \theta / \ln \eta>0$ goes to 0 as $\eta \rightarrow 1$, so $\beta \rightarrow 0$ as $\varrho \rightarrow 0$. Therefore,

$$
\eta^{n}\left|\left(P_{c_{0}}^{n}\right)^{\prime}\left(\zeta_{1}\right)\right|^{-1} \leq\left|\left(P_{c_{0}}^{n}\right)^{\prime}\left(\zeta_{1}\right)\right|^{-(1-\beta)} \leq K_{3} \varrho^{(1-\beta) d \alpha},
$$

where $K_{3}=\left(A_{0} d^{-1}\right)^{-(1-\beta)}$. By the recursive formula for $r_{\zeta_{1}}$, as in the proof of the previous lemma we have

$$
r_{\zeta_{1}} \leq K_{3} \varrho^{(1-\beta) d \alpha} R \varrho^{1 / d}+4 \varrho\left(K_{2}+K_{3} \varrho^{(1-\beta) d \alpha}\right) .
$$

Since by definition $d \alpha>1-1 / d$ we have $1 / d+(1-\beta) d \alpha>1$ if $\varrho$ is small enough. Thus we may suppose that $\varrho$ is close enough to 0 so that $r_{\zeta_{1}} \leq 8 K_{2} \varrho$. This is the assertion with $K_{1}=8 K_{2}$.

3. If $\left|\zeta_{1}-c_{0}\right| \leq 2\left(K_{1}+4\right) \varrho$ then $B_{r_{\zeta_{1}}+4 \varrho}\left(\zeta_{1}\right) \subset B_{4\left(K_{1}+4\right) \varrho}\left(c_{0}\right)$ so

$$
P_{c_{0}}^{-1}\left(B_{r_{\zeta_{1}}+4 \varrho}\left(\zeta_{1}\right)\right) \subset B_{\left(4\left(K_{1}+4\right) \varrho\right)^{1 / d}}(0) .
$$

Moreover $|\zeta| \leq\left(2\left(K_{1}+4\right)\right)^{1 / d}$, thus if $R \geq 2\left(4\left(K_{1}+4\right)\right)^{1 / d}$ the lemma follows in this case.

If $\left|\zeta_{1}-c_{0}\right|>2\left(K_{1}+4\right) \varrho$ then the corresponding inverse branch of $P_{c_{0}}^{-1}$ defined in $B_{\zeta_{1}+4 \varrho}\left(\zeta_{1}\right)$ is univalent and has distortion bounded by some constant $K_{4}>0$, given by the Koebe Distortion Theorem. Hence, if $W$ is the connected component of $P_{c_{0}}^{-1}\left(B_{r_{\zeta_{1}}+4 \varrho}\left(\zeta_{1}\right)\right)$ that contains $\zeta$ then

but

$$
W \subset B_{K_{4}\left|P_{c_{0}}^{\prime}(\zeta)\right|^{-1}\left(r_{\zeta_{1}}+4 \varrho\right)}(\zeta),
$$

$$
\begin{aligned}
K_{4}\left|P_{c_{0}}^{\prime}(\zeta)\right|^{-1}\left(r_{\zeta_{1}}+4 \varrho\right) & \leq K_{4} d\left(2\left(K_{1}+4\right) \varrho\right)^{-(d-1) / d}\left(K_{1}+4\right) \varrho \\
& =K_{4} d 2^{-1}\left(2\left(K_{1}+4\right) \varrho\right)^{1 / d} .
\end{aligned}
$$

Thus the lemma holds with

$$
R=\max \left(K_{4} d 2^{-1}\left(2\left(K_{1}+4\right)\right)^{1 / d}, 2\left(4\left(K_{1}+4\right)\right)^{1 / d}\right) .
$$

Proof of the Main Lemma. Let $\left\{z_{i}\right\}$ be a $\varrho$-dense set in $V$ and let $r_{i}=$ $\sup _{\zeta \in B_{\rho}\left(z_{i}\right)} r_{\zeta}$ where the supremum is over all $\zeta$ for which $r_{\zeta}$ is defined. Put $D_{i}=B_{r_{i}+2 \varrho}\left(z_{i}\right)$. By Lemma 3.3 there is a constant $M>0$ only depending on $P_{c_{0}}$ such that $\operatorname{diam}\left(D_{i}\right) \leq M \varrho^{1 / d}$.

Since $\left\{z_{i}\right\}$ is $\varrho$-dense in $V$, for all $z \in J_{c_{0}}$ there is $z_{i}$ at a distance at most $\varrho$ from $z$ and therefore $B_{\varrho}(z) \subset B_{2 \varrho}\left(z_{i}\right) \subset D_{i}$.

Fix $i$ and let $\zeta_{k}$ be a convergent sequence in $B_{\varrho}\left(z_{i}\right)$ such that $r_{\zeta_{k}} \rightarrow r_{i}$ as $k \rightarrow \infty$. Moreover choose a convergent sequence of preimages $\zeta_{k}^{\prime}$ of $\zeta_{k}$ under $P_{c_{0}}$. Taking a subsequence if necessary we may assume that there is $z_{j}$ such that $\left|\zeta_{k}^{\prime}-z_{j}\right| \leq \varrho$, so $r_{\zeta_{k}^{\prime}} \leq r_{j}$. By Lemma 3.4 if $W_{k}$ is the connected component of $P_{c_{0}}^{-1}\left(B_{r_{\zeta_{k}}+4 \varrho}\left(\zeta_{k}\right)\right)$ that contains $\zeta_{k}^{\prime}$ then

$$
W_{k} \subset B_{\zeta_{\zeta_{k}^{\prime}}}\left(\zeta_{k}^{\prime}\right) \subset D_{j}
$$

Since $B_{\varrho}\left(D_{i}\right) \subset \bigcup_{k} B_{r_{\zeta_{k}}+4 \varrho}\left(\zeta_{k}\right)$, the lemma follows. 
4. Similarities between dynamical and parameter planes. In this section we prove Theorems B and C. Fix a semihyperbolic parameter $c_{0} \in \partial \mathcal{M}_{d}$ throughout this section. In this section the positive constants $C_{0}, C_{1}, \ldots$ and all implicit constants depend on $P_{c_{0}}$ only.

Consider a Markov partition $U_{a}, a \in A$, for $\omega(0)$ as in Section 2. Recall that $l>1$ is the least integer such that $P_{c_{0}}^{l}(0) \in \omega(0)$. Let $z_{0}=P_{c_{0}}^{l}(0)$. We will denote by $U_{n}$ the $n$th step piece of the Markov partition that contains $z_{0}$. By the Univalent Extension Property and Koebe Distortion Theorem it follows that $\operatorname{dist}\left(z_{0}, U_{n}\right) \sim \operatorname{diam}\left(U_{n}\right)$; see Section 2.2. Denote by $V_{n}$ the pull-back of $U_{n}$ to 0 by $P_{c_{0}}^{l}$. Then we have

$$
\operatorname{dist}\left(\partial V_{n}, 0\right) \sim \operatorname{diam}\left(V_{n}\right) \sim\left(\operatorname{diam}\left(U_{n}\right)\right)^{1 / d} .
$$

It follows by the Markov property and considering that the $U_{a}$ are puzzles that if $W$ is a pull-back of $V_{n}$, then either $W \cap V_{n}=\emptyset$ or $W \subset V_{n}$. We define

$$
K_{n}=\left\{z \mid P_{c_{0}}^{k}(z) \notin V_{n} \text { for } k \geq 0\right\},
$$

which is a closed and forward invariant set. It follows that $V_{n}$ is the connected component of $\mathbb{C}-K_{n}$ that contains 0 and for every connected component $W$ of $\mathbb{C}-K_{n}$ there is $k$ so that $P_{c_{0}}^{k}: W \rightarrow V_{n}$ is a biholomorphism. Let us consider the following easy lemma about the geometry of $K_{n}$.

Lemma 4.1. There is a constant $C_{1}>0$ only depending on $P_{c_{0}}$ such that for $n$ large and any connected component $W$ of $\mathbb{C}-K_{n}$,

(i) $\operatorname{diam}(W) \leq C_{1} \operatorname{diam}\left(V_{n}\right)$.

(ii) $\operatorname{diam}(W) \leq C_{1} \operatorname{dist}\left(W, c_{0}\right) \operatorname{diam}\left(V_{n}\right)$.

Proof. Consider the constants $\varepsilon>0, \theta \in(0,1)$ and $C>0$ given by [CJY]; see Preliminaries. Choose $n$ large enough so that $V_{n} \subset B_{\varepsilon / 2}(0)$. Thus, if $m \geq 0$ is such that $P_{c_{0}}^{m}: W \rightarrow V_{n}$ is a biholomorphism, the corresponding pull-back $W_{0}$ of $B_{\varepsilon}(0)$ is univalent and moreover $c_{0} \notin W_{0}$. Denote by $W^{\prime}$ the corresponding pull-back of $B_{\varepsilon / 2}(0)$. So by the Koebe Distortion Theorem there is a constant $D>0$ so that $\operatorname{diam}(W) \leq D \operatorname{diam}\left(W^{\prime}\right) \operatorname{diam}\left(V_{n}\right) \leq$ $D C \theta^{m} \operatorname{diam}\left(V_{n}\right)$.

Hence (i) follows for $C_{1} \geq D C$. On the other hand, note that the modulus of the annulus $W_{0}-\overline{W^{\prime}}$ is equal to the modulus of the annulus $B_{\varepsilon}-\overline{B_{\varepsilon / 2}(0)}$, which does not depend on $\varepsilon$. Since $c_{0} \notin W_{0}$, there is a universal constant $K_{0}>0$ so that

$$
\operatorname{diam}\left(W^{\prime}\right) \leq K_{0} \operatorname{dist}\left(W^{\prime}, c_{0}\right) \leq K_{0} \operatorname{dist}\left(W, c_{0}\right),
$$

and so (ii) follows.

4.1. Holomorphic motions compatible with dynamics. Recall that a holomorphic motion $j$ of a set $X \subset \mathbb{C}$ defined in an open set $W \subset \mathbb{C}$ is a map 
$j: W \times X \rightarrow \mathbb{C}$ so that for all $\lambda \in W$, the map $j_{\lambda}: X \rightarrow \mathbb{C}$ is injective and for every $x \in X, j_{\lambda}(x)$ depends holomorphically on $\lambda$.

Proposition 4.2. Let $V$ be the neighborhood of $J_{c_{0}}$ bounded by the equipotential with potential 1, as in the Main Lemma. Then there are constants $\nu>0$ and $C_{2}>0$, only depending on $P_{c_{0}}$, such that for large $n$, there exists a holomorphic motion $i_{n}: B_{n} \times K_{n} \rightarrow \mathbb{C}$, where $B_{n}=B_{\nu \operatorname{diam}\left(U_{n}\right)}\left(c_{0}\right)$, such that:

(i) $\left(i_{n}\right)_{c_{0}}$ is the identity.

(ii) $i_{n}$ is compatible with dynamics, that is, $\left(i_{n}\right)_{c}\left(P_{c_{0}}(z)\right)=P_{c}\left(\left(i_{n}\right)_{c}(z)\right)$ for all $(c, z) \in B_{n} \times K_{n}$.

(iii) For $(c, z) \in B_{n} \times\left(K_{n} \cap V\right)$ we have $\left|\left(i_{n}\right)_{c}(z)-z\right| \leq C_{2} \operatorname{diam}\left(V_{n}\right)$. Moreover, if $(c, z) \in B_{n} \times K_{n}$ is such that $c=\left(i_{n}\right)_{c}(z)$, then $c=\varphi_{\mathcal{M}_{d}}^{-1} \circ \varphi_{c_{0}}(z)$.

Before proving this proposition let us deduce Theorem $\mathrm{C}$ from it.

Proof of Theorem $C$. We will prove that $d_{\mathrm{H}}\left(J_{c_{0}}, K_{c}\right) \leq C_{3}\left|c-c_{0}\right|^{1 / d}$ for some constant $C_{3}>0$ only depending on $P_{c_{0}}$. The assertion about $d_{\mathrm{H}}\left(J_{c_{0}}, J_{c}\right)$ follows in a similar way. Consider $n$ large and let $c \in B_{n}-B_{n+1}$ be so that $\left|c-c_{0}\right| \sim \operatorname{diam}\left(U_{n}\right)$. By Proposition 4.2 we have

$$
d_{\mathrm{H}}\left(J_{c_{0}},\left(i_{n}\right)_{c}\left(K_{n} \cap J_{c_{0}}\right)\right) \leq C_{4}\left|c-c_{0}\right|^{1 / d} .
$$

Moreover by Lemma 4.1 every connected component of $\mathbb{C}-\left(i_{n}\right)_{c}\left(K_{n}\right)$ has diameter less than $C_{5}\left|c-c_{0}\right|^{1 / d}$. Hence $\left(i_{n}\right)_{c}\left(K_{n} \cap J_{c_{0}}\right) \subset K_{c}$ is $C_{5}\left|c-c_{0}\right|^{1 / d_{-}}$ dense in $K_{c}$. Therefore $d_{\mathrm{H}}\left(J_{c_{0}}, K_{c}\right) \leq\left(C_{4}+C_{5}\right)\left|c-c_{0}\right|^{1 / d}$ as wanted.

Proof of Proposition 4.2.

1. Put $V^{\prime}=P_{c_{0}}^{-1}(V)$ and let $\delta>0$ be so small that for all $c \in B_{\delta}\left(c_{0}\right)$, the function $\varphi_{c}^{-1}$ is well defined in $\{\ln |\zeta| \geq 1 / d\}$; so the holomorphic motion $i: B_{\delta}\left(c_{0}\right) \times\left(\mathbb{C}-V^{\prime}\right) \rightarrow \mathbb{C}$ defined by $i_{c}=\varphi_{c}^{-1} \circ \varphi_{c_{0}}$ has properties (i) and (ii).

2. Note that there is a constant $\mu_{d}>0$ only depending on $d$ so that if $D \subset \mathbb{C}$ satisfies $\operatorname{diam}(D)<\mu_{d} \operatorname{dist}(0, D)$, then $P_{c_{0}}$ is univalent in $D$. Let $n$ be large and recall that $\operatorname{dist}\left(0, \partial V_{n}\right) \sim \operatorname{diam}\left(V_{n}\right) \sim\left(\operatorname{diam}\left(U_{n}\right)\right)^{1 / d}$. Then there is $\nu_{0}>0$ only depending on $P_{c_{0}}$ such that if we take $\varrho=\varrho_{n}=\nu_{0} \operatorname{diam}\left(U_{n}\right)$ in the Main Lemma, then the $D_{i}$ (given by the Main Lemma) intersecting $K_{n}$ satisfy $\operatorname{diam}\left(D_{i}\right)<\mu_{d} \operatorname{dist}\left(D_{i}, 0\right)$ and therefore $P_{c_{0}}$ is injective in such $D_{i}$.

Note that for $w \in V-V^{\prime}$ and $c \in B_{\delta}\left(c_{0}\right), i_{c}(w)$ is bounded independently of $c$ and $w$. Thus there is a constant $C_{6}>0$ only depending on $P_{c_{0}}$ such that for every $w \in V-V^{\prime}$ and $c \in B_{\delta}\left(c_{0}\right)$ we have $\left|i_{c}(w)-w\right| \leq C_{6} \delta$. Hence, by Schwarz' Lemma, for all $w \in V-V^{\prime}$ and all $c \in B_{\min \left(\varrho_{n}, \delta\right)}(0)$ we have $\left|i_{c}(w)-w\right| \leq C_{6} \varrho_{n}$. So we may choose $\nu \in\left(0, \nu_{0}\right)$, only depending on $P_{c_{0}}$, so that $\left|i_{c}(w)-w\right| \leq \varrho_{n}$ for all $c \in B_{\nu \operatorname{diam}\left(U_{n}\right)}\left(c_{0}\right)=B_{n}$ and $w \in V-V^{\prime}$. 
3. We define $i_{n}$ to be equal to $i$ in $B_{n} \times\left(\mathbb{C}-V^{\prime}\right)$. Let $z \in K_{n}-J_{c_{0}}$ be such that $z \in V^{\prime}$ and let $k$ be such that $P_{c_{0}}^{k}(z) \in V-V^{\prime}$. Moreover let $c \in B_{n}=B_{\nu \operatorname{diam}\left(U_{n}\right)}\left(c_{0}\right)$.

By the above, $\left|i_{c}\left(P_{c_{0}}^{k}(z)\right)-P_{c_{0}}^{k}(z)\right| \leq \varrho_{n}$. So by the Main Lemma there is $D_{i}$ so that $i_{c}\left(P_{c_{0}}^{k}(z)\right) \in D_{i}$ and there is $D_{j}$ containing the connected component $W$ of $P_{c_{0}}^{-1}\left(B_{\varrho_{n}}\left(D_{i}\right)\right)$ that contains $P_{c_{0}}^{k-1}(z)$. Moreover, since $c \in$ $B_{n} \subset B_{\varrho_{n}}\left(c_{0}\right)$, we have $P_{c}(W) \subset B_{\varrho_{n}}\left(P_{c_{0}}(W)\right)$ for $c \in B_{n}$. So it follows that there is a unique preimage of $i_{c}\left(P_{c_{0}}^{k}(z)\right)$ under $P_{c}$ in $D_{j}$.

Repeating this process we obtain a sequence $i_{j}$, for $0 \leq j \leq k$, such that $P_{c_{0}}^{j}(z) \in D_{i_{j}}$ and a uniquely determined orbit $z_{0}, \ldots, z_{k}=i_{c}\left(P_{c_{0}}^{k}(z)\right)$ of $P_{c}$ such that $z_{j} \in D_{i_{j}}$. We define $\left(i_{n}\right)_{c}(z)=z_{0}$, which clearly depends holomorphically on $c$ and satisfies (i) and (ii).

Let us prove that $\left(i_{n}\right)_{c}$ is injective in $K_{n}-J_{c_{0}}$, so that $i_{n}$ is a holomorphic motion of $K_{n}-J_{c_{0}}$ defined in $B_{n}$. If not we would have different $w_{0}, w_{1} \in$ $K_{n}$ such that for some $c \in B_{n},\left(i_{n}\right)_{c}\left(w_{0}\right)=\left(i_{n}\right)_{c}\left(w_{1}\right)$. Considering that $\left(i_{n}\right)_{c_{0}}=$ id we must have $i_{c}\left(w_{0}\right)=i_{c}\left(w_{1}\right)=0$, since $\left(i_{n}\right)_{c}$ is compatible with dynamics. But this is not possible since $\left(i_{n}\right)_{c}\left(w_{0}\right)$ is contained is some $D_{i}$ intersecting $K_{n}$ and by hypothesis we have $\operatorname{dist}\left(D_{i}, 0\right) \geq \mu_{d} \operatorname{diam}\left(D_{i}\right)>0$.

So $i_{n}: B_{n} \times\left(K_{n}-J_{c_{0}}\right) \rightarrow \mathbb{C}$ is a holomorphic motion. Since $V_{n}$ is a puzzle we may suppose that for any $z \in K_{n} \cap J_{c_{0}}$ there is a ray contained in $K_{n}$ landing at $z$. So $\overline{K_{n}-J_{c_{0}}}=K_{n}$ and by the $\lambda$-Lemma of [MSS], the holomorphic motion $i_{n}$ extends to $K_{n}$.

4. It remains to prove that if $(c, z) \in B_{n} \times\left(K_{n}-J_{c_{0}}\right)$ is such that $c=\left(i_{n}\right)_{c}(z)$, then $c=\varphi_{\mathcal{M}_{d}}^{-1} \circ \varphi_{c_{0}}(z)$. Let $\zeta=\varphi_{c_{0}}(z)$. Since $V_{n}$ is a puzzle we may suppose that the piece of ray $\mathcal{R}=\left\{\varphi_{c_{0}}^{-1}(r \zeta) \mid r>1\right\}$ is contained in $K_{n}$, so $\left(i_{n}\right)_{c}(\mathcal{R})$ is a piece of ray for $P_{c}$ with the same angle as $\mathcal{R}$ and by construction the potential of $c=\left(i_{n}\right)_{c}(z)$ for $P_{c}$ is the same as that of $z$ for $P_{c_{0}}$. Thus $\varphi_{\mathcal{M}_{d}}(c)=\varphi_{c}(c)=\varphi_{c_{0}}(z)$.

4.2. Conformality of external maps. In this section we prove Theorem B about the $C^{1+1 / d}$-conformality of the map $\varphi_{\mathcal{M}_{d}}^{-1} \circ \varphi_{c_{0}}$ and its inverse at $c_{0}$. The proof depends on Lemma 4.3 below. We also prove the sharpness of Theorem $\mathrm{C}$ at the end of this section.

Lemma 4.3. Let $n$ be large and let $B_{n}=B_{\nu \operatorname{diam}\left(U_{n}\right)}\left(c_{0}\right)$ be as in Proposition 4.2. Then there is a constant $C_{7}>0$ only depending on $P_{c_{0}}$ such that for $S>1$ given and $w \in K_{n}$ such that $S^{-1} \operatorname{diam}\left(U_{n}\right) \leq\left|w-c_{0}\right| \leq S \operatorname{diam}\left(U_{n}\right)$ we have, for $n \geq N_{0}(S)$ and $c \in B_{n}$,

$$
\left|\left(i_{n}\right)_{c}(w)-w-\left(z(c)-c_{0}\right)\right| \leq C_{7}\left(\operatorname{diam}\left(U_{n}\right)\right)^{1+1 / d} .
$$

Proof. 1. By the expansive property of hyperbolic sets there is $\varepsilon_{0}>0$ such that for any $w$ close to $c_{0}$ there is $N=N(w)>0$ such that 


$$
\left|P_{c_{0}}^{N}(w)-P_{c_{0}}^{N}\left(c_{0}\right)\right|>\varepsilon_{0} .
$$

For fixed $w$, consider the smallest such $N$. Reducing $\varepsilon_{0}$ if necessary we may assume that

$$
\varepsilon_{0}<\left|P_{c_{0}}^{N}(w)-P_{c_{0}}^{N}\left(c_{0}\right)\right|<\varepsilon_{0} D \ll \min _{a \in A} \operatorname{diam}\left(U_{a}\right)
$$

where $D$ is the supremum of $\left|P_{c_{0}}^{\prime}(z)\right|$ over $z \in \bigcup_{a \in A} U_{a}$. By the Bounded Distortion Property,

$$
\left|\left(P_{c_{0}}^{N}\right)^{\prime}\left(c_{0}\right)\right| \cdot\left|w-c_{0}\right| \geq K^{-1}\left|P_{c_{0}}^{N}(w)-P_{c_{0}}^{N}\left(c_{0}\right)\right| \geq K^{-1} \varepsilon_{0} .
$$

So $\left|\left(P_{c_{0}}^{N}\right)^{\prime}\left(c_{0}\right)\right|^{-1} \leq K \varepsilon_{0}^{-1}\left|w-c_{0}\right| \leq K \varepsilon_{0}^{-1} S \varrho_{n}$, where $\varrho_{n}=\operatorname{diam}\left(U_{n}\right)$.

2. Consider the holomorphic motion $j: B_{\delta}\left(c_{0}\right) \times \bigcup_{a \in A} U_{a} \rightarrow \mathbb{C}$ given by Proposition 2.3 and let $R>0$ be such that $j\left(B_{\delta}\left(c_{0}\right) \times \bigcup_{a \in A} U_{a}\right) \subset B_{R}(0)$; see Section 2.2.

Fix $c \in B_{n}$ and let $w^{\prime}$ be such that $j_{c}\left(w^{\prime}\right)=\left(i_{n}\right)_{c}(w)$, so $j_{c}\left(P_{c_{0}}^{N}\left(w^{\prime}\right)\right)=$ $\left(i_{n}\right)_{c}\left(P_{c_{0}}^{N}(w)\right)$. By Schwarz' Lemma applied to the function $\widehat{c} \mapsto j_{\widehat{c}}\left(P_{c_{0}}^{N}\left(w^{\prime}\right)\right)$ we have

$$
\left|P_{c_{0}}^{N}\left(w^{\prime}\right)-\left(i_{n}\right)_{c}\left(P_{c_{0}}^{N}(w)\right)\right| \leq C_{8}\left|c-c_{0}\right| \leq C_{8} \nu \varrho_{n}
$$

where $C_{8}=2 R / \delta$. By Proposition 4.2,

$$
\left|\left(i_{n}\right)_{c}\left(P_{c_{0}}^{N}(w)\right)-P_{c_{0}}^{N}(w)\right| \leq C_{9} \varrho_{n}^{1 / d}
$$

so for large $n$ we have $\left|P_{c_{0}}^{N}\left(w^{\prime}\right)-P_{c_{0}}^{N}(w)\right| \leq C_{9} \varrho_{n}^{1 / d}+C_{8} \nu \varrho_{n} \leq 2 C_{9} \varrho_{n}^{1 / d}$ and by the Bounded Distortion Property,

$$
\left|w^{\prime}-w\right| \leq K\left|\left(P_{c_{0}}^{N}\right)^{\prime}\left(c_{0}\right)\right|^{-1}\left|P_{c_{0}}^{N}\left(w^{\prime}\right)-P_{c_{0}}^{N}(w)\right| \leq K^{2} \varepsilon_{0}^{-1} 2 C_{9} S \varrho_{n}^{1+1 / d} .
$$

So, if $n$ is larger than some $N_{0}$ (depending on $S$ ), we have $(2 S)^{-1} \varrho_{n} \leq$ $\left|w^{\prime}-c_{0}\right| \leq 2 S \varrho_{n}$.

3. By 2 we have

$$
\left|c-c_{0}\right|\left(\ln \left(\frac{1}{2 R}\left|w^{\prime}-c_{0}\right|\right)\right)^{-1} \leq C_{10} \varrho_{n}\left(\ln \left(S \varrho_{n}\right)\right)^{-1} \rightarrow 0 \quad \text { as } n \rightarrow \infty .
$$

Considering that $j_{c}\left(w^{\prime}\right)=\left(i_{n}\right)_{c}(w)$ we have, by Lemma 2.4,

$$
\begin{aligned}
\left|w^{\prime}-j_{c}\left(w^{\prime}\right)-\left(c_{0}-j_{c}\left(c_{0}\right)\right)\right| & \leq 4 \frac{\left|c-c_{0}\right|}{\operatorname{diam}(B)}\left|w^{\prime}-c_{0}\right|\left(\ln \left(\frac{1}{2 R}\left|w^{\prime}-c_{0}\right|\right)\right)^{-1} \\
& \leq \frac{8}{\operatorname{diam}(B)} C_{10} S \varrho_{n}^{2}\left(\ln \left(S \varrho_{n}\right)\right)^{-1}
\end{aligned}
$$

Since $j_{c}\left(w^{\prime}\right)=\left(i_{n}\right)_{c}(w)$ and $j_{c}\left(c_{0}\right)=\left(i_{n}\right)_{c}\left(c_{0}\right)$, it follows that for $n$ large,

$$
\begin{aligned}
\mid w- & \left(i_{n}\right)_{c}(w)-\left(c_{0}-\left(i_{n}\right)_{c}\left(c_{0}\right)\right) \mid \\
& \leq K^{2} \varepsilon_{0}^{-1} 2 C_{9} S \varrho_{n}^{1+1 / d}+\frac{8 C_{10}}{\operatorname{diam}(B)} S \varrho_{n}^{2}\left(\ln \left(S \varrho_{n}\right)\right)^{-1} \leq C_{7} S \varrho_{n}^{1+1 / d} .
\end{aligned}
$$




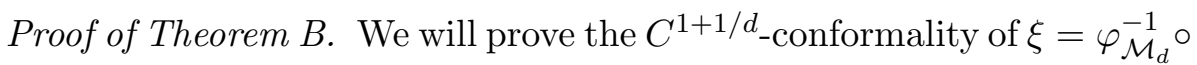
$\varphi_{c_{0}}$ at $c_{0}$; the conformality of its inverse follows in the same way. Note that $\xi$ is defined in $\mathbb{C}-J_{c_{0}}$.

Let $z(c)=j_{c}\left(c_{0}\right)$ be the dynamical continuation of the critical value $c_{0}$, so that $1-z^{\prime}\left(c_{0}\right) \neq 0$; see Section 2.2 and Appendix 2. Since $c-z(c)=$ $\lambda\left(c-c_{0}\right)+\mathcal{O}\left(\left|c-c_{0}\right|^{2}\right)$, it is enough to prove that for $w \notin J_{c_{0}}$ close to $c_{0}$ we have

$$
\left|w-c_{0}-(\xi(w)-z(\xi(w)))\right|=\mathcal{O}\left(\left|w-c_{0}\right|^{1+1 / d}\right) .
$$

Fix $w \notin J_{c_{0}}$ close to $c_{0}$ and let $c^{\prime}$ close to $c_{0}$ be such that $w=c_{0}+c^{\prime}-z\left(c^{\prime}\right)$, so $\left|w-c_{0}\right|=\left|c^{\prime}-z\left(c^{\prime}\right)\right| \sim\left|c^{\prime}-c_{0}\right|$. Let $n$ be the greatest integer such that $c^{\prime} \in B_{n+1}$, so $\operatorname{diam}\left(U_{n}\right) \sim\left|c^{\prime}-c_{0}\right|$. Given $C>0$, to be chosen below, let $\eta:[0,1] \rightarrow \mathbb{C}$ be defined by

$$
\eta(\theta)=c^{\prime}+C\left|c^{\prime}-c_{0}\right|^{1+1 / d} e^{2 \pi i \theta} .
$$

Then there are two cases.

Case 1: $w \in K_{n}$. By Lemma 4.3, for all $\widetilde{c} \in B_{n}$ we have

$$
\left|\left(i_{n}\right) \tilde{c}(w)-w-\left(z(\widetilde{c})-c_{0}\right)\right|=\mathcal{O}\left(\left|c^{\prime}-c_{0}\right|^{1+1 / d}\right) .
$$

We may suppose that $w$ is close enough to $c_{0}$ so that $\eta(\theta) \in B_{n}$ for all $\theta \in[0,1]$. Note that $\left|w-c_{0}-(\widetilde{c}-z(\widetilde{c}))\right|=\left|c^{\prime}-z\left(c^{\prime}\right)-(\widetilde{c}-z(\widetilde{c}))\right| \sim\left|c^{\prime}-\widetilde{c}\right|$. So we may choose $C>0$ large enough so that for all $\theta \in[0,1]$,

$$
\left|\left(i_{n}\right)_{\eta(\theta)}(w)-w-\left(z(\eta(\theta))-c_{0}\right)\right|<\left|w-c_{0}-(\eta(\theta)-z(\eta(\theta)))\right| .
$$

Thus by the Rouché theorem there is $c \in B_{n}$ such that $\left(i_{n}\right)_{c}(w)=c$ and $\left|c-c^{\prime}\right| \leq C\left|c^{\prime}-c_{0}\right|^{1+1 / d}$. It follows that $c=\xi(w)$ and

$$
\left|w-c_{0}-(c-z(c))\right|=\left|c^{\prime}-z\left(c^{\prime}\right)-(c-z(c))\right|=\mathcal{O}\left(\left|w-c_{0}\right|^{1+1 / d}\right) .
$$

CASE 2: $w \notin K_{n}$. Let $y \in \partial K_{n}$ be the unique point which is in the same ray as $w$ and let $l$ be the piece of ray joining them. Let $U$ be the connected component of $\mathbb{C}-K_{n}$ containing $w$. By Lemma 4.1, $\operatorname{diam}(U)=$ $\mathcal{O}\left(\left|y-c_{0}\right|^{1+1 / d}\right)$, therefore $|y-w|=\mathcal{O}\left(\left|y-c_{0}\right|^{1+1 / d}\right)$ and $\left|y-c_{0}\right| \sim\left|w-c_{0}\right| \sim$ $\left|c^{\prime}-c_{0}\right|$. So $\operatorname{diam}(U)=\mathcal{O}\left(\left|c^{\prime}-c_{0}\right|^{1+1 / d}\right)$.

By [St], $i_{n}$ extends to a continuous function in $B_{n} \times \mathbb{C}$ such that for each $\widetilde{c} \in B_{n}$, the $\left(i_{n}\right)_{\tilde{c}}$ is a homeomorphism of $\mathbb{C}$. Then it follows from the last observation and from Lemma 4.3 that, for $\widetilde{c} \in B_{n}$,

$$
\operatorname{diam}\left(\left(i_{n}\right)_{\tilde{c}}(U)\right)=\mathcal{O}\left(\left|c^{\prime}-c_{0}\right|^{1+1 / d}\right) .
$$

Given $\widetilde{c} \in B_{n}$ and $w_{0} \in \varphi_{\widetilde{c}}^{-1}\left(\varphi_{c_{0}}(w)\right)$ consider the Green line $\widetilde{l}$ joining $w_{0}$ and $\left(i_{n}\right)_{\tilde{c}}(y)$. Then $\tilde{l} \cap K_{n}=\left\{i_{\tilde{c}}(y)\right\}$. It follows that $\widetilde{l} \subset\left(i_{n}\right)_{\tilde{c}}(U)$, so $w_{0} \in i_{\tilde{c}}(U)$ and $\varphi_{c}^{-1}\left(\varphi_{c_{0}}(w)\right) \subset i_{\widetilde{c}}(U)$.

Therefore, for all $\widetilde{c} \in B_{n}$ and $\zeta \in \varphi_{\widetilde{c}}^{-1}\left(\varphi_{c_{0}}(w)\right)$ we have $\left|\zeta-i_{\widetilde{c}}(y)\right| \leq$ $\operatorname{diam}\left(i_{\widetilde{c}}(U)\right)=\mathcal{O}\left(\left|c^{\prime}-c_{0}\right|^{1+1 / d}\right)$. Thus, by the considerations of Case 1 ap- 
plied to $y$ instead of $w$, we may choose the constant $C>0$ large enough so that

$$
\left|\zeta-w-\left(z(\eta(\theta))-c_{0}\right)\right|<\left|w-c_{0}-(\eta(\theta)-z(\eta(\theta)))\right|
$$

for all $\zeta \in \varphi_{\eta(\theta)}^{-1}\left(\varphi_{c_{0}}(w)\right)$ and $\theta \in[0,1]$. Then by the Rouché theorem there is $c$ such that $\varphi_{\mathcal{M}_{d}}(c)=\varphi_{c}(c)=\varphi_{c_{0}}(w)$ and $\left|c-c^{\prime}\right| \leq C\left|c^{\prime}-c_{0}\right|^{1+1 / d}$. It follows that $c=\xi(w)$ and

$$
\left|w-c_{0}-(c-z(c))\right|=\left|c^{\prime}-z\left(c^{\prime}\right)-(c-z(c))\right|=\mathcal{O}\left(\left|w-c_{0}\right|^{1+1 / d}\right) .
$$

Similarity factor. Recall that $\lambda=1-z^{\prime}\left(c_{0}\right) \neq 0$; see Appendix 2. For $n \geq 0$ let $z_{n}(c)=P_{c}^{n}(z(c))$, which is equal to $j_{c}\left(P_{c}^{n}(z(c))\right)$ for $n \geq l$. Since the image of the holomorphic motion $j$ is bounded, it follows by Schwarz' Lemma that there is $D>0$ such that $\left|z_{n}^{\prime}\left(c_{0}\right)\right| \leq D$ for $n \geq 0$. By the equation $z_{n+1}(c)=z_{n}(c)^{d}+c$ we have

$$
z_{n+1}^{\prime}(c)=d z_{n}(c)^{d-1} z_{n}^{\prime}(c)+1=P_{c}^{\prime}\left(z_{n}(c)\right) z_{n}^{\prime}(c)+1 .
$$

Thus, for $n \geq 0$ we have

$$
z_{0}^{\prime}(c)=-\left(\frac{1}{P_{c}^{\prime}\left(z_{0}(c)\right)}+\frac{1}{\left(P_{c}^{2}\right)^{\prime}\left(z_{0}(c)\right)}+\ldots+\frac{1}{\left(P_{c}^{n}\right)^{\prime}\left(z_{0}(c)\right)}\right)+\frac{z_{n}^{\prime}(c)}{\left(P_{c}^{n}\right)^{\prime}\left(z_{0}(c)\right)} .
$$

Considering that $z=z_{0}, z\left(c_{0}\right)=c_{0}$ and that $\left(P_{c_{0}}^{n}\right)^{\prime}\left(c_{0}\right) \rightarrow \infty$ as $n \rightarrow \infty$, we have

$$
\lambda=1-z^{\prime}\left(c_{0}\right)=\sum_{n \geq 0} \frac{1}{\left(P_{c_{0}}^{n}\right)^{\prime}\left(c_{0}\right)} .
$$

Sharpness of Theorem $C$. Now we can prove that the estimate of Theorem $\mathrm{C}$ is sharp. We will use the fact that $J_{c_{0}}$ is a John domain; see [CJY]. This means that there is $\delta>0$ such that for any ray $\mathcal{R}$ landing at a point $z \in J_{c_{0}}$ and any $w \in \mathcal{R}$ the ball $B_{\delta|w-z|}(w)$ is disjoint from $J_{c_{0}}$.

Let $z(c)=j_{c}\left(c_{0}\right)$ be the dynamical continuation of the critical value $c_{0}$, so that $z^{\prime}\left(c_{0}\right) \neq 0$; see Section 2.2 and Appendix 2. In particular, we have $\left|z(c)-c_{0}\right| \sim\left|c-c_{0}\right|$. Hence, if $w \in P_{c}^{-1}(z(c))$ then $|w| \sim\left|c-c_{0}\right|^{1 / d}$. One may choose $c$ such that $w$ belongs to the ray of $J_{c_{0}}$ landing at 0 , so by the John $\operatorname{property} \operatorname{dist}\left(w, J_{c_{0}}\right) \sim\left|c-c_{0}\right|^{1 / d}$. But $z(c) \in J_{c}$ so $w \in J_{c}$, and therefore $d_{\mathrm{H}}\left(J_{c_{0}}, J_{c}\right) \sim\left|c-c_{0}\right|^{1 / d}$.

5. Hausdorff dimension. In this section we prove Theorem A that follows easily from Theorem B and the HD Lemma stated below. This lemma is a criterion for the convergence of the Hausdorff dimension of Julia sets, and follows from a similar lemma in [BR].

In this section the positive constants $C_{1}, C_{2}, \ldots$ and all implicit constants depend on $P_{c_{0}}$ only. 
HD LEMMA. Let $c_{0} \in \partial \mathcal{M}_{d}$ be such that $P_{c_{0}}$ is semihyperbolic and consider a sequence $c_{n} \rightarrow c_{0}$ such that

$$
\left|c_{n}-c_{0}\right|^{d /(d-1)}\left(\operatorname{dist}\left(c_{n}, J_{c_{n}}\right)\right)^{-1} \rightarrow 0 .
$$

Then $\operatorname{HD}\left(J_{c_{n}}\right) \rightarrow \operatorname{HD}\left(J_{c_{0}}\right)$.

The proof of this lemma is in Section 5.1. The proof of Theorem A is based on the following lemma.

LEMMA 5.1. Let $c_{0} \in \partial \mathcal{M}_{d}$ be such that $P_{c_{0}}$ is semihyperbolic and let $c$ be close to $c_{0}$. Then there is a constant $C>0$, only depending on $P_{c_{0}}$, such that

if $\operatorname{dist}\left(c, \mathcal{M}_{d}\right) \geq C\left|c-c_{0}\right|^{1+1 / d}, \quad$ then $\operatorname{dist}\left(c, J_{c}\right) \geq\left|c-c_{0}\right|^{1+1 / d}$.

Proof. Let $c \in \mathbb{C}$ be such that $\operatorname{dist}\left(c, \mathcal{M}_{d}\right)>C\left|c-c_{0}\right|^{1+1 / d}$ for some constant $C>0$ to be determined later, and let $z=\varphi_{c_{0}}^{-1} \circ \varphi_{\mathcal{M}_{d}}(c)$. For $m \gg 1$ let $B_{m}=B_{\nu \operatorname{diam}\left(U_{m}\right)}\left(c_{0}\right)$ and $i_{m}: B_{m} \times K_{m} \rightarrow \mathbb{C}$ be as in Proposition 4.2. Let $m$ be such that $c \in B_{m}-B_{m+1}$. By Theorem B there is $S>1$, only depending on $P_{c_{0}}$, such that $S^{-1} \operatorname{diam}\left(U_{m}\right)<\left|z-c_{0}\right|<S \operatorname{diam}\left(U_{m}\right)$.

If $C>0$ is large enough there is $C^{\prime}>0$ so that $\operatorname{dist}\left(z, \partial K_{m}\right)>$ $C^{\prime}\left|z-c_{0}\right|^{1+1 / d}$ (cf. Lemma 4.1). Moreover we may choose $C^{\prime}>0$ arbitrarily large, by choosing $C$ large.

Consider $w$ such that $|w-z|=C^{\prime}\left|z-c_{0}\right|^{1+1 / d}$. Thus $w \in K_{m}-J_{c_{0}}$. Moreover, if $c$ is close enough to $c_{0}$, then $(2 S)^{-1} \operatorname{diam}\left(U_{m}\right)<\left|w-c_{0}\right|<$ $2 S \operatorname{diam}\left(U_{m}\right)$. Let $C_{7}>0$ be the constant given by Lemma 4.3 . Since $c=$ $\left(i_{m}\right)_{c}(z)$, for any $\zeta \in B_{\left|c-c_{0}\right|^{1+1 / d}}(c)$ we have

$$
\begin{aligned}
\left|\left(i_{m}\right)_{c}(w)-\zeta\right| \geq & \left|\left(i_{m}\right)_{c}(w)-c\right|-|c-\zeta| \\
\geq & |w-z|-\left|c-c_{0}\right|^{1+1 / d} \\
& \quad-\left|\left(i_{m}\right)_{c}(z)-z(c)-\left(z-c_{0}\right)\right|-\left|\left(i_{m}\right)_{c}(w)-z(c)-\left(w-c_{0}\right)\right| .
\end{aligned}
$$

By Lemma 4.3 the last two quantities do not exceed $2 C_{7} S\left(\operatorname{diam}\left(U_{m}\right)\right)^{1+1 / d}$. Since $c \in B_{m}$, we have $\left|c-c_{0}\right| \leq \nu \operatorname{diam}\left(U_{m}\right)$. Thus, choosing $C>0$ large enough so that $C^{\prime}>4 C_{7} S+\nu^{1+1 / d}$, we have $\left|\left(i_{m}\right)_{c}(w)-\zeta\right|>0$.

Since this holds for every $w$ such that $|w-z|=C^{\prime}\left|z-c_{0}\right|^{1+1 / d}$, it follows by the Rouché theorem that there is $w_{\zeta}$ such that $\left|w_{\zeta}-z\right|<$ $C^{\prime}\left|z-c_{0}\right|^{1+1 / d}$ and $\left(i_{m}\right)_{c}\left(w_{\zeta}\right)=\zeta$. As remarked above $w_{\zeta} \notin J_{c_{0}}$ in this case, so $\zeta=\left(i_{m}\right)_{c}\left(w_{\zeta}\right) \notin J_{c}$. Thus $B_{\left|c-c_{0}\right|^{1+1 / d}}(c) \cap J_{c}=\emptyset$.

Proof of Theorem $A$. Let $C>0$ be as in the previous lemma and suppose that the sequence $c_{n} \rightarrow c$ is such that $\operatorname{dist}\left(c_{n}, \mathcal{M}_{d}\right) \geq C\left|c_{n}-c_{0}\right|^{1+1 / d}$. By the previous lemma $\operatorname{dist}\left(c_{n}, J_{c_{n}}\right) \geq\left|c_{n}-c_{0}\right|^{1+1 / d}$. Thus,

$$
\left|c_{n}-c_{0}\right|^{d /(d-1)}\left(\operatorname{dist}\left(c_{n}, J_{c_{n}}\right)\right)^{-1} \leq\left|c_{n}-c_{0}\right|^{1 /(d(d-1))} \rightarrow 0
$$

as $n \rightarrow \infty$. Hence, by the $\operatorname{HD}$ Lemma, $\operatorname{HD}\left(J_{c_{n}}\right) \rightarrow \operatorname{HD}\left(J_{c_{0}}\right)$. 
5.1. Conformal measures and atoms. The proof of the HD Lemma is as follows. For $c \in \mathbb{C}-\mathcal{M}_{d}$ there is a unique conformal probability measure $\mu_{c}$ for $P_{c}$ supported in $J_{c}$. Moreover $\mu_{c}$ has exponent $d_{c}=\operatorname{HD}\left(J_{c}\right)$; see [Su]. This means that for every measurable set $U$ where $P_{c}$ is injective, $\mu_{c}\left(P_{c}(U)\right)=\int_{U}\left|P_{c}^{\prime}\right|^{d_{c}} d \mu_{c}$. Furthermore the $\mu_{c}$ measure of a point is zero, that is, $\mu_{c}$ is not atomic.

The unique conformal probability measure for $P_{c_{0}}$, supported in $J_{c_{0}}$, either has exponent $d_{c_{0}}=\operatorname{HD}\left(J_{c_{0}}\right)$ or is atomic, supported in $\left\{P_{c_{0}}^{-n}(0)\right\}_{n \geq 0}$; see $[\mathrm{DU}]$ and $[\mathrm{McM}]$. Thus to prove that

$$
\lim _{n \rightarrow \infty} \operatorname{HD}\left(J_{c_{n}}\right)=\operatorname{HD}\left(J_{c_{0}}\right)
$$

it is enough to prove that

$$
\lim _{r \rightarrow 0} \lim _{n \rightarrow \infty} \mu_{c_{n}}\left(B_{r}(0)\right)=0 .
$$

In fact, if $\mu_{c_{0}}$ is any weak limit of $\left\{\mu_{c_{n}}\right\}_{n \geq 1}$, then $\mu_{c_{0}}$ is a conformal probability measure supported in $J_{c_{0}}$. The previous limit implies that the measure $\mu_{c_{0}}$ is not atomic at 0 , so it has exponent $d_{c_{0}}$ and it follows that $d_{c_{n}} \rightarrow d_{c_{0}}$; see also [McM], [DSZ] and [UZ].

Consider a Markov partition $U_{a}, a \in A$, as in Section 2 and consider a holomorphic motion $j: B_{\delta}\left(c_{0}\right) \times \bigcup_{a \in A} U_{a} \rightarrow \mathbb{C}$ given by Proposition 2.3. Taking $\delta>0$ smaller if necessary we may assume that there are constants $C_{0}>0$ and $\theta_{0} \in(0,1)$ such that for all $m \geq 1$, all $c \in B_{\delta}\left(c_{0}\right)$ and all $w \in i_{c}(\omega(0))$, we have $\left|\left(P_{c}^{m}\right)^{\prime}(w)\right|^{-1} \leq C_{0} \theta_{0}^{m}$. Moreover we may suppose that there is a uniform Bounded Distortion Property: There is a constant $K>1$ so that for every $c \in B_{\delta}\left(c_{0}\right)$, every $k \geq 1$ and every $k$ th step piece $W$ of the Markov partition $j_{c}\left(U_{a}\right), a \in A$, the distortion of $P_{c}^{k}$ in $W$ is bounded by $K$; cf. Section 2.2 .

Recall that $U_{n}$ is the $n$th step piece containing $P_{c_{0}}^{l}(0) \in \omega(0)$ and $V_{n}$ is the pull-back of $U_{n}$ by $P_{c 0}^{l}$ containing 0 . Denote $j_{c}\left(U_{n}\right)$ by $U_{n}^{c}$ and let $V_{n}^{c}$ be the pull-back of $U_{n}^{c}$ by $P_{c}^{l}$ containing 0. It follows that for $r>0$ small there is $n=n(r) \rightarrow \infty$ as $r \rightarrow 0$ so that $B_{r}(0) \subset V_{n}^{c}$ for all $c$ sufficiently close $c_{0}$. Hence it is enough to prove that

$$
\lim _{n \rightarrow \infty} \lim _{s \rightarrow \infty} \mu_{c_{s}}\left(V_{n}^{c_{s}}\right)=0
$$

Proof of HD Lemma. 1. Let $D$ be a disc containing 0, small enough so that for $c \in B_{\delta}\left(c_{0}\right),\left.P_{c}^{l}\right|_{D}$ is at most of degree $d$. Refining the Markov partition if necessary, suppose that $U_{1}^{c} \subset P_{c}^{l}(D)$ for all $c \in B_{\delta}\left(c_{0}\right)$.

Since for $c \notin \mathcal{M}_{d}$ the probability measure $\mu_{c}$ is not atomic, for all $n \geq 1$ we have

$$
\mu_{c}\left(V_{n}^{c}\right)=\sum_{m \geq n} \mu_{c}\left(V_{m}^{c}-V_{m+1}^{c}\right)
$$


Recall that $z(c)=j_{c}\left(c_{0}\right)$ is the dynamical continuation of the critical value $c_{0}$ and $z^{\prime}\left(c_{0}\right) \neq 1$; see Appendix 2. For $c \in B_{\delta}\left(c_{0}\right)$ let $\zeta(c)=$ $j_{c}\left(P_{c_{0}}^{l-1}\left(c_{0}\right)\right)=P_{c}^{l-1}(z(c))$ and put $q_{c}=P_{c}^{l}(0)$. Note that for $m \geq 1$ we have

$$
\mu_{c}\left(V_{m}-V_{m}^{c}\right) \leq d \mu_{c}\left(U_{m}^{c}-U_{m+1}^{c}\right) \inf _{\left(V_{m}^{c}-V_{m+1}^{c}\right) \cap J_{c}}\left|\left(P_{c}^{l}\right)^{\prime}(z)\right|^{-d_{c}} .
$$

By the uniform Bounded Distortion Property and considering that $\mu_{c}$ is a probability measure, we have

$$
\mu_{c}\left(U_{m}^{c}-U_{m+1}^{c}\right) \leq K^{d_{c}}\left|\left(P_{c}^{m}\right)^{\prime}(\zeta(c))\right|^{-d_{c}} .
$$

On the other hand there is $C_{1}>0$ such that for all $c \in B_{\delta}\left(c_{0}\right)$ and $z \in V_{1}^{c}$,

$$
\left|\left(P_{c}^{l}\right)^{\prime}(z)\right|>C_{1}\left|P_{c}^{l}(z)-q_{c}\right|^{(d-1) / d} .
$$

2. Let $k=k(c)$ be the greatest integer such that $q_{c} \in U_{k}^{c}$; see Figure 2. Let $m \geq 1$. Then there are three cases.

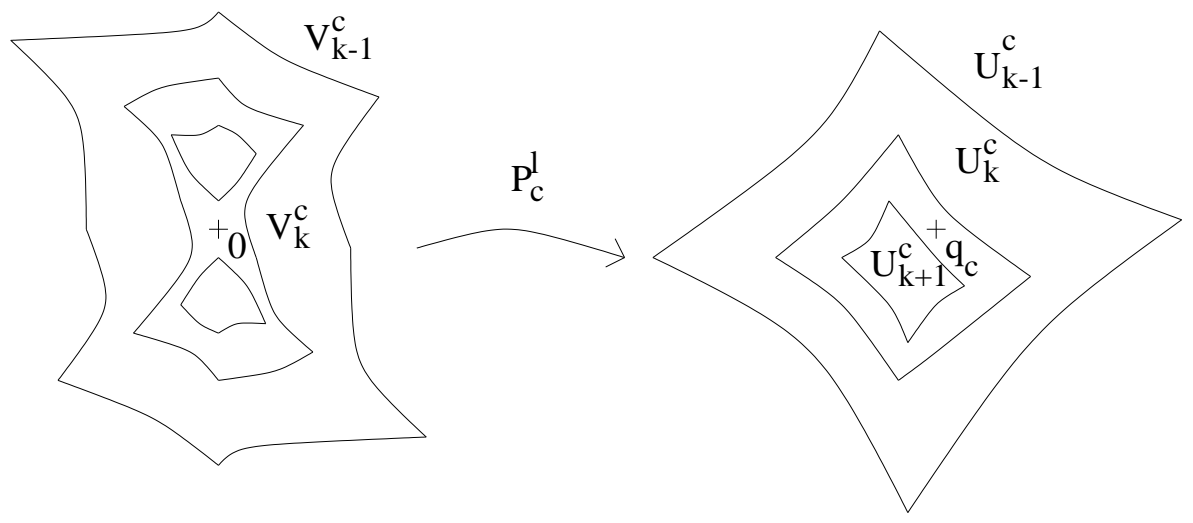

Fig. 2. Relative position of $q_{c}=i_{c}\left(P_{c_{0}}^{l}(0)\right)$ in the Markov partition

CASE 1: $k-1 \leq m \leq k+1$. By the uniform Bounded Distortion Property and by Transversality (Appendix 2), we have

$$
\left|\left(P_{c}^{m}\right)^{\prime}(\zeta(c))\right|^{-1} \sim\left|\zeta(c)-q_{c}\right| \sim|z(c)-c| \sim\left|c-c_{0}\right|,
$$

with implicit constants independent of $c \in B_{\delta}\left(c_{0}\right)$. Hence $\left|\left(P_{c}^{m}\right)^{\prime}(\zeta(c))\right|^{-1} \leq$ $C_{2}\left|c-c_{0}\right|$ for some $C_{2}>0$ independent of $c$. On the other hand

$$
\operatorname{dist}\left(q_{c},\left(U_{m}^{c}-U_{m+1}^{c}\right) \cap J_{c}\right) \geq \operatorname{dist}\left(q_{c}, J_{c}\right) \geq C_{3} \operatorname{dist}\left(c, J_{c}\right) .
$$

So for all $z \in V_{m}^{c}-V_{m+1}^{c} \cap J_{c}$

$$
\left|\left(P_{c}^{l}\right)^{\prime}(z)\right|>C_{1} C_{3}^{(d-1) / d}\left(\operatorname{dist}\left(c, J_{c}\right)\right)^{(d-1) / d},
$$

thus

$$
\mu_{c}\left(V_{m}-V_{m+1}\right) \leq C_{4}\left|c-c_{0}\right|^{d_{c}}\left(\operatorname{dist}\left(c, J_{c}\right)\right)^{-d_{c}(d-1) / d},
$$

where $C_{4}=d\left(K C_{2}\left(C_{1} C_{3}^{(d-1) / d}\right)^{-1}\right)^{d_{c}}$. 
CAse 2: $m<k-1$. Note that

$$
\operatorname{dist}\left(q_{c}, U_{m}^{c}-U_{m+1}^{c}\right) \geq \operatorname{dist}\left(\partial U_{m+1}^{c}, U_{m+2}^{c}\right),
$$

thus by the uniform Bounded Distortion Property,

$$
\operatorname{dist}\left(q_{c}, U_{m}^{c}-U_{m+1}^{c}\right)>C_{5}\left|\left(P_{c}^{m}\right)^{\prime}(\zeta(c))\right|^{-1} .
$$

Hence, by 1 we have

$$
\begin{aligned}
\left|\left(P_{c}^{l}\right)^{\prime}(z)\right| & >C_{1}\left(\operatorname{dist}\left(q_{c}, U_{m}^{c}-U_{m+1}^{c}\right)\right)^{(d-1) / d} \\
& \geq C_{1} C_{5}^{(d-1) / d}\left|\left(P_{c}^{m}\right)^{\prime}(\zeta(c))\right|^{-(d-1) / d} .
\end{aligned}
$$

Therefore,

$\mu_{c}\left(V_{m}^{c}-V_{m+1}^{c}\right) \leq d K^{d_{c}}\left|\left(P_{c}^{m}\right)^{\prime}(\zeta(c))\right|^{-d_{c}}\left(C_{1} C_{5}^{(d-1) / d}\right)^{-d_{c}}\left|\left(P_{c}^{m}\right)^{\prime}(\zeta(c))\right|^{d_{c}(d-1) / d}$. Thus $\mu_{c}\left(V_{m}^{c}-V_{m+1}^{c}\right) \leq C_{6} \theta_{0}^{m d_{c} / d}$, where $C_{6}=d K^{d_{c}}\left(C_{1} C_{5}^{(d-1) / d}\right)^{-d_{c}} C_{0}^{d_{c} / d}$.

CASE 3: $m>k+1$. We have $\operatorname{dist}\left(q_{c}, U_{m}^{c}-U_{m+1}^{c}\right) \geq \operatorname{dist}\left(\partial U_{m-1}^{c}, U_{m}^{c}\right)$. Thus reducing $C_{5}>0$ if necessary, we have, as in Case 2,

$$
\operatorname{dist}\left(q_{c}, U_{m}^{c}-U_{m+1}^{c}\right)>C_{5}\left|\left(P_{c}^{m}\right)^{\prime}(\zeta(c))\right|^{-1},
$$

and $\mu_{c}\left(V_{m}^{c}-V_{m+1}^{c}\right) \leq C_{6} \theta_{0}^{m d_{c} / d}$.

3. By 1 and 2 , for $n \geq 1$ we have

$$
\mu_{c}\left(V_{n}^{c}\right) \leq 3 C_{4}\left|c-c_{0}\right|^{d_{c}}\left(\operatorname{dist}\left(c, J_{c}\right)\right)^{-d_{c}(d-1) / d}+C_{6} \sum_{m \geq n, m \neq k-1, k, k+1} \theta_{0}^{m d_{c} / d} .
$$

Since

$$
\sum_{m \geq n} \theta_{0}^{m d_{c} / d}=\frac{\left(\theta_{0}^{d_{c}} / d\right)^{n}}{1-\theta^{d_{c} / d}}
$$

and since by hypothesis $\left|c_{s}-c_{0}\right|\left(\operatorname{dist}\left(c_{s}, J_{c_{s}}\right)\right)^{-(d-1) / d} \rightarrow 0$ as $s \rightarrow \infty$, we conclude that

$$
\lim _{n \rightarrow \infty} \lim _{s \rightarrow \infty} \mu_{c_{s}}\left(V_{n}^{c_{s}}\right)=0
$$

\section{Appendix 1. Proof of corollaries of Theorem B}

Proof of asymptotic similarity. We only use the conformality of $\varphi_{\mathcal{M}_{d}}^{-1} \circ \varphi_{c_{0}}$ at $c_{0}$ and that $J_{c_{0}}$ has empty interior. Put $\xi=\varphi_{\mathcal{M}_{d}}^{-1} \circ \varphi_{c_{0}}: \overline{\mathbb{C}}-J_{c_{0}} \rightarrow \overline{\mathbb{C}}-\mathcal{M}_{d}$, which is a proper map.

Let $w \in J_{c_{0}}$ and put $r=\left|w-c_{0}\right|$. Consider a sequence $\left\{w_{i}\right\}$ disjoint from $J_{c_{0}}$ such that $w_{i} \rightarrow w$. Since $\xi$ is proper we may suppose that $c_{i}=\xi\left(w_{i}\right) \rightarrow$ $c \in \mathcal{M}_{d}$. Thus, by Theorem B there is $C_{1}>0$ only depending on $P_{c_{0}}$ such that $\left|w-c_{0}-\lambda\left(c-c_{0}\right)\right| \leq C_{1} r^{1+1 / d}$.

Let $c \in \mathcal{M}_{d}$ be close to $c_{0}$ and put $r=\left|c-c_{0}\right|$. By the Rouché theorem and by Theorem $\mathrm{B}$ there is $C_{2}>0$, only depending on $P_{c_{0}}$, such that if $w$ satisfies $\left|w-c_{0}\right| \sim r$ and $B_{C_{2} r^{1+1 / d}}(w) \cap J_{c_{0}}=\emptyset$, then $c^{\prime}=c_{0}+\lambda^{-1}\left(w-c_{0}\right) \notin$ 
$\mathcal{M}_{d}$. Thus, if $w=c_{0}+\lambda\left(c-c_{0}\right)$, then $B_{C_{2} r^{1+1 / d}}(w) \cap J_{c_{0}} \neq \emptyset$. Let $w^{\prime} \in$ $B_{C_{2} r^{1+1 / d}}(w) \cap J_{c_{0}}$. By Theorem B there is $C_{3}>0$, only depending on $P_{c_{0}}$, such that $\left|c-c_{0}-\lambda\left(w^{\prime}-c_{0}\right)\right| \leq C_{3} r^{1+1 / d}$. Then the corollary follows.

Proof of the second corollary. It follows by [Ma] that $P_{c_{0}}$ is uniformly expanding in the set of accumulation points of the orbit of 0 , denoted by $\omega(0)$. So the Bounded Distortion Property holds: for $\delta>0$ small and $z \in \omega(0)$ there is $n \geq 0$ so that $P_{c_{0}}^{n}\left(B_{r}(z)\right)$ is of unit size and the distortion of $P_{c_{0}}^{n}$ in $B_{\delta}(z)$ is bounded by some constant independent of $z$; see Section 2 .

By [DU] for all $z \in J_{c_{0}}$ and $r>0$ small $m_{D}\left(B_{r}(z)\right) \sim r^{D}$, where $m_{D}$ denotes the restriction to $J_{c_{0}}$ of the $D$-dimensional Hausdorff measure. Hence $J_{c_{0}}$ can be covered with a collection of $\sim r^{-D / d}$ balls of radius $r^{1 / d}$. By the Bounded Distortion Property $\left(J_{c_{0}}-c_{0}\right)_{r}$ can also be covered by such a collection of balls and by the previous corollary this also holds for $\left(\mathcal{M}_{d}-c_{0}\right)_{r}$. Hence the measure of $\left(\mathcal{M}_{d}-c_{0}\right)_{r}$ is $\mathcal{O}\left(r^{-D / d} r^{2 / d}\right)$, and therefore the measure of $\mathcal{M}_{d} \cap B_{r}\left(c_{0}\right)$ is $\mathcal{O}\left(r^{2+(2-D) / d}\right)$.

Appendix 2. Transversality. Fix $d \geq 2$ and consider a semihyperbolic polynomial $P_{c_{0}}(z)=z^{d}+c$ such that $c_{0} \in \partial \mathcal{M}_{d}$. By [Ma], $P_{c_{0}}$ is uniformly expanding in $\omega(0)$. So for some $\delta>0$ there is a holomorphic motion $j$ : $B_{\delta}\left(c_{0}\right) \times \omega(0) \rightarrow \mathbb{C}$ which is compatible with dynamics; see [Sh]. By the expansive property of hyperbolic sets there is $l>1$ such that $P_{c_{0}}^{l}(0) \in \omega(0)$, so there is a holomorphic function $z(c)$ such that $z\left(c_{0}\right)=c_{0}$ and $P_{c}^{l-1}(z(c))=$ $j_{c}\left(P_{c}^{l-1}\left(c_{0}\right)\right)$ for $c \in B_{\delta}\left(c_{0}\right)$.

The objective of this appendix is to prove the following property.

Transversality. The graph of the function $z$ is transversal to the diagonal at $\left(c_{0}, c_{0}\right)$; that is, $z\left(c_{0}\right)=c_{0}$ and $z^{\prime}\left(c_{0}\right) \neq 1$.

In the Misiurewicz case, this property is well known and there are various different proofs of this fact. For example there is an algebraic proof by A. Gleason in [DH2] and there is a proof of A. Epstein from a more abstract result (in $[\mathrm{E}]$ ) based on infinitesimal Thurston rigidity.

We prove Transversality in the more general semihyperbolic case, using Thurston rigidity. For $d=2$ this also follows from [vS], which was done independently. The idea is to argue by contradiction. So if Transversality does not hold, then one can find two different parameters $c_{1}$ and $c_{2}$, close to $c_{0}$, such that their respective polynomials have the same dynamical properties and then we prove that in fact $c_{1}=c_{2}$. More concretely it will be proved that the polynomials $P_{c_{1}}$ and $P_{c_{2}}$ are equivalent in the sense of Thurston; see [DH3]. This implies that $P_{c_{1}}$ and $P_{c_{2}}$ are holomorphically conjugate and since $c_{1}$ and $c_{2}$ are close to $c_{0}$ it follows that $c_{1}=c_{2}$. One may also argue with external rays, but with Thurston rigidity the argument generalizes to 
rational functions. I am grateful to A. Douady who suggested to me to argue by contradiction.

Let us consider a Markov partition $U_{a}, a \in A$, as in Section 2 and note that as in Section 4.2 we may suppose that $j$ is defined in $\bigcup_{a \in A} U_{a}$. Denote by $K_{c_{0}}$ the maximal invariant set for the Markov partition. Enlarging it if necessary, we may suppose that $P_{c_{0}}^{l}(0)$ is an accumulation point of periodic points in $K_{c_{0}}$.

Proof of Transversality. Suppose that Transversality does not hold. By [DH2] we have $z(c) \not \equiv c$, therefore there is $m>1$ such that $|z(c)-c| \sim$ $\left|c-c_{0}\right|^{m}$. Let $w$ be close to $c_{0}$ so that $P_{c_{0}}^{l-1}(w) \in K_{c_{0}}$ and let $c_{1}^{\prime} \neq c_{2}^{\prime}$ be such that $c_{i}^{\prime}-z\left(c_{i}^{\prime}\right)+c_{0}=w$ for $i=1,2$. Thus, if $w$ is close enough to $c_{0}$ then

$$
\frac{\left|c_{1}^{\prime}-c_{2}^{\prime}\right|}{\left|c_{1}^{\prime}-c_{0}\right|}>\frac{1}{2}\left|e^{2 \pi i / m}-1\right| .
$$

Applying the Rouché theorem as in the proof of Theorem B (with the help of Lemma 4.3) there are $c_{1} \neq c_{2}$ such that $P_{c_{i}}^{l-1}\left(c_{i}\right)=j_{c_{i}}\left(P_{c_{0}}^{l-1}(w)\right)$ with $c_{i} \in D$ close to $c_{0}$ for $i=1,2$; see also Lemma 5.1.

1. Put $U^{\prime}=\bigcup_{a \in A} \bar{U}_{a}$ and let $U_{\infty}^{\prime}$ be a neighborhood of $\infty$ such that $\overline{U^{\prime}}$ and $\overline{U_{\infty}^{\prime}}$ are disjoint.

Let $D$ be a small disc centered at $c_{0}$ and let $i: D \times\left(\overline{U^{\prime}} \cup \overline{U_{\infty}^{\prime}}\right) \rightarrow \mathbb{C}$ be the holomorphic motion that coincides with $j$ in $U^{\prime}$ and is defined by $i_{c}(z)=\varphi_{c}^{-1} \circ \varphi_{c_{0}}(z)$ for $z \in \overline{U_{\infty}^{\prime}}$. So $i$ is compatible with dynamics.

By hypothesis $P_{c_{0}}^{l-1}\left(c_{0}\right)$ is an accumulation point of periodic points in $K_{c_{0}}$, so $c_{0}$ is the limit of a sequence of preperiodic points $w$ with $P_{c_{0}}^{l-1}(w) \in$ $K_{c_{0}}$. Extend $i$ to $\bigcup_{0<m<l} P_{c_{0}}^{m}(w)$ so that $i$ is compatible with dynamics. Let $c_{1}$ and $c_{2}$ be the parameters corresponding to $w$ as above, so that $i_{c_{i}}(w)=c_{i}$.

Put $V^{\prime}=U^{\prime} \cup U_{\infty}^{\prime} \cup \bigcup_{0<m<l} P_{c_{0}}^{m}(w)$ and $V=P_{c_{0}}^{-1}\left(V^{\prime}\right)$. Extend $i$ to $\bar{V}-\{0\}$ by dynamics and let $i_{c}(0) \equiv 0$. Since $D$ is conformally equivalent to a disc, one can extend $i$ to $D \times \overline{\mathbb{C}}$; see $[\mathrm{Sł}]$.

2. For $c \in D$ consider the homeomorphism $\theta_{c}^{\prime}=i_{c} \circ i_{c_{1}}^{-1}$ of $\overline{\mathbb{C}}$, so that $\theta_{c_{1}}^{\prime} \equiv$ id. For $z \in \bar{V}-\{0\}$ let $\theta_{c}(z)=j_{c}^{-1} \circ j_{c_{1}}(z)$, so $P_{c} \circ \theta_{c}=\theta_{c}^{\prime} \circ P_{c_{1}}(z)$ in $i_{c_{1}}(\bar{V})$ and $\theta_{c}$ coincides with $\theta_{c}^{\prime}$ in $i_{c_{1}}\left(K_{c_{0}}\right)$ and in $i_{c_{1}}\left(\bigcup_{0<m<l} P_{c_{0}}^{m}(w)\right)$.

Choose $\delta>0$ such that $\left\{\left|z-c_{0}\right|<\delta\right\} \cap i_{c}\left(\overline{V^{\prime}}\right)=i_{c}(w)$ for $c \in D$; such a $\delta$ can be chosen before choosing $w$. For $c \in D$ let $h_{c}$ be a homeomorphism of $\overline{\mathbb{C}}$ depending continuously on $c$ so that $h_{c}$ is the identity outside the ball $\left\{\left|z-c_{0}\right|<\delta\right\}$ and $h_{c}(c)=i_{c}(w)$. So $h_{c_{i}}\left(c_{i}\right)=c_{i}$ for $i=1,2$. Moreover we assume that $h_{c_{i}}$ is the identity for $i=1,2$.

Then for $c \in D$ we see that $h_{c} \circ P_{c}: \overline{\mathbb{C}}-\bar{V} \rightarrow \overline{\mathbb{C}}-\overline{V^{\prime}}$ is a $d$-fold covering map. Since $h_{c} \circ P_{c} \circ \theta_{c} \equiv \theta_{c}^{\prime} \circ P_{c_{1}}$ in $i_{c_{1}}(\bar{V})$, there is a unique way to extend $\theta_{c}$ to a homeomorphism so that $h_{c} \circ P_{c} \circ \theta_{c} \equiv \theta_{c}^{\prime} \circ P_{c_{1}}$. 
3. Considering that $h_{c_{i}}$ is the identity for $i=1,2$, we have $P_{c_{2}} \circ \theta_{c_{2}} \equiv$ $\theta_{c_{2}}^{\prime} \circ P_{c_{1}}$. Moreover $\theta_{c_{1}}=\theta_{c_{1}}^{\prime}=$ id and for all $c \in D$, the homeomorphism $\theta_{c}^{-1} \circ \theta_{c}^{\prime}$ is the identity on $K_{c_{0}} \cup i_{c_{1}}\left(\bigcup_{0<m<l} P_{c_{0}}^{m}(w)\right)$. So $\theta_{c}^{-1} \circ \theta_{c}^{\prime}$ is the identity in the post-critical set of $P_{c_{1}}$.

Consider a path $\gamma:[0,1] \rightarrow D$ such that $\gamma(0)=c_{1}$ and $\gamma(1)=c_{2}$. Then $t \mapsto \theta_{\gamma(t)}^{-1} \circ \theta_{\gamma(t)}^{\prime}$ is an isotopy between id and $\theta_{c_{2}}^{-1} \circ \theta_{c_{2}}^{\prime}$, relative to the post-critical set of $P_{c_{1}}$. This means exactly that $P_{c_{1}}$ and $P_{c_{2}}$ are equivalent in the sense of Thurston. Therefore $P_{c_{1}}$ and $P_{c_{2}}$ are holomorphically conjugate; see [DH3]. Since $c_{1}$ and $c_{2}$ are close to $c_{0}$ we find that $c_{1}=c_{2}$, which is a contradiction.

\section{References}

[BR] J. Barragan and J. Rivera-Letelier, manuscript, 1998.

[CG] L. Carleson and T. Gamelin, Complex Dynamics, Springer, 1993.

[CJY] L. Carleson, P. Jones and J.-C. Yoccoz, Julia and John, Bol. Soc. Brasil. Mat. 25 (1994), 1-30.

[DU] M. Denker and M. Urbański, On Hausdorff measures on Julia sets of subexpanding rational maps, Israel J. Math. 76 (1992), 193-224.

[D] A. Douady, Does a Julia set depend continuously on the polynomial?, in: R. Devaney (ed.), Complex Dynamical Systems, Proc. Sympos. Appl. Math. 49, Amer. Math. Soc., 1994, 91-138.

[DH1] A. Douady et J. Hubbard, Etude dynamique des polynômes complexes, Publ. Math. Orsay 84-02 (1984) (première partie) and 84-04 (1985) (deuxième partie).

[DH2] - - - On the dynamics of polynomial-like mappings, Ann. Sci. École Norm. Sup. 18 (1985), 287-344.

[DH3] - - - A proof of Thurston's topological characterization of rational functions, Acta Math. 171 (1993), 263-297.

[DSZ] A. Douady, P. Sentenac et M. Zinsmeister, Implosion parabolique et dimension de Hausdorff, C. R. Acad. Sci. Paris Sér. I Math. 325 (1997), 765-772.

[E] A. Epstein, Towers of finite type complex analytic maps, thesis, CUNY, 1993.

[GSm] J. Graczyk and S. Smirnov, Weak expansion and geometry of Julia sets, manuscript, 1999.

[GSw] J. Graczyk and G. Świątek, Harmonic measure and expansion on the boundary of the connectedness locus, Invent. Math. 142 (2000), 605-629.

[H] J. Hubbard, Local connectivity of Julia sets and bifurcation loci: three theorems of J.-C. Yoccoz, in: L. R. Goldberg and A. V. Phillips (eds.), Topological Methods in Modern Mathematics, Publish or Perish, 1993, 457-511.

[K] J. Kiwi, Rational rays and critical portraits of complex polynomials, preprint IMS at Stony Brook, \#1997/15.

[L] T. Lei, Similarity between the Mandelbrot set and Julia sets, Comm. Math. Phys. 134 (1990), 587-617.

[Ma] R. Mañé, On a lemma of Fatou, Bol. Soc. Brasil. Mat. 24 (1993), 1-12.

[MSS] R. Mañé, P. Sad and D. Sullivan, On the dynamics of rational maps, Ann. Sci. École Norm. Sup. 16 (1983), 193-217.

[McM] C. McMullen, Hausdorff dimension and conformal dynamics II: Geometrically finite rational maps, Comment. Math. Helv. 75 (2000), 535-593. 
[R-L1] J. Rivera-Letelier, Similarities between the Mandelbrot set and Julia sets, preliminary version, 1999.

[R-L2] —, Rational maps with decay of geometry: rigidity, Thurston's algorithm and local connectivity, preprint IMS at Stony Brook, \#2000/9.

[R-L3] -, Rigid annuli, Thurston's pull-back argument and the Collet-Eckmann condition, preliminary version, 1999.

[R] D. Ruelle, Repellers for real analytic maps, Ergodic Theory Dynam. Systems 2 (1982), 99-107.

[Sh] M. Shishikura, The Hausdorff dimension of the boundary of the Mandelbrot set and Julia sets, Ann. of Math. 147 (1998), 225-267.

[Sł] Z. Słodkowski, Holomorphic motions and convex hulls, Proc. Amer. Math. Soc. 111 (1991), 347-355.

[vS] S. van Strien, Misiurewicz maps unfold generically (even if they are critically non-finite), Fund. Math. 163 (2000), 39-54.

[Su] D. Sullivan, Conformal dynamical systems, in: Geometric Dynamics (Rio de Janeiro, 1981), Lecture Notes in Math. 1007, Springer, Berlin, 1983, 725-752.

[Th] W. P. Thurston, On the combinatorics of iterated rational maps, manuscript, 1985.

[U] M. Urbański, Rational functions with no recurrent critical points, Ergodic Theory Dynam. Systems 14 (1994), 391-414.

[UZ] M. Urbański and M. Zinsmeister, Continuity of Hausdorff dimension of the Julia-Lavaurs sets as a function of the phase, preprint, 2000.

[W] L. Wenstorm, Parameter scaling for the Fibonacci point, preprint IMS at Stony Brook, \#1996/4.

Institute for Mathematical Sciences

SUNY at Stony Brook

Stony Brook, NY 11794-3660, U.S.A.

E-mail: rivera@math.sunysb.edu
Universidad Católica del Norte Avenida Angamos 0610 Antofagasta, Chile

Received 15 June 2000;

in revised form 29 August 2001 